Full Paper

\title{
The Electronic Structure of the Nitrogen Atoms of Allyl (5- pyridin-2-yl-[1,3,4]-thiadiazol-2-yl)-amine
}

\section{Leokadia Strzemecka}

Department of Organic Chemistry, Faculty of Pharmacy, Medical Academy of Lublin, Staszica 6, 20-081 Lublin, Poland; e-mail: leokadia.strzemecka@am.lublin.pl; fax: +48-81 5328903

Received: 28 February 2006; in revised form: 18 May 2006 / Accepted: 20 May 2006 / Published: 28 June 2006

\begin{abstract}
The resonance structures of allyl-(5-pyridin-2-yl-[1,3,4]-thiadiazol-2-yl)-amine have been determined by means of its ${ }^{1} \mathrm{H}-(100 \mathrm{MHz}, 500 \mathrm{MHz}){ }^{13} \mathrm{C}$ - and ${ }^{15} \mathrm{~N}-\mathrm{NMR}$ spectra and B3LYP/6-31G* computations. The tautomeric equilibrium of this compound has been observed in the ${ }^{1} \mathrm{H}-\mathrm{NMR}$ spectra (100 MHz).
\end{abstract}

Keywords: Allyl-(5-pyridin-2-yl-[1,3,4]-thiadiazol-2-yl)-amine; electronic structure, tautomerism

\section{Introduction}

1,3,4-Thiadiazoles bearing an amino group at the C2 atom can exist in different tautomeric forms (Figure 1). The tautomeric equilibrium is influenced by the exocyclic N6 and C5 substituents on the 1,3,4-thiadiazole ring [1, 2]. 2-Amino-[1,3,4]-thiadiazole exists in the amino form in solution and in the solid state. The same tautomer is the main form in 5-alkoxy derivatives. In the case of a 2-hydrazino substituent the hydrogen atom also appears at the exocyclic N6 nitrogen atom, but in the sulfonamido group the tautomeric equilibrium shifts towards the imino form (type b, Figure 1). It therefore appeared of interest to examine the tautomeric equilibrium of (5-pyridin-2-yl-[1,3,4] thiadiazol-2-yl)-amine derivatives bearing allyl- and (3-phenylallyl)- substituents (compounds $\mathbf{1}$ and 2, respectively). Earlier $100 \mathrm{MHz}{ }^{1} \mathrm{H}-\mathrm{NMR}$ studies in the solution of 1-acyl-(aroyl-)-4-(3-phenylallyl)- 
thiosemicarbazides [3] and $\mathrm{N}^{1}$-[(3-phenylallyl)-thiocarbamyl]amidrazones [4] as well as of the products obtained of the result of the acid cyclization of the linear compounds [5,6] confirmed various tautomeric structures for the above mentioned compounds and the transformation products. Allyl- (1) and 3-phenylallyl-(5-pyridin-2-yl-[1,3,4]thiadiazol-2-yl)-amine (2) [6, 7], another product of the acid cyclization of the linear $\mathrm{N}^{1}$-[ allyl-(3-phenylallyl)-thiocarbamyl]-amidrazone compounds, turned out to be suitable to study the tautomeric transformations.

Figure 1. Tautomers a and $\mathbf{b}$ of allyl- (1) and 3-phenylallyl-(5-pyridin-2-yl- [1,3,4]thiadiazol-2-yl)-amine (2), with atom numbering.

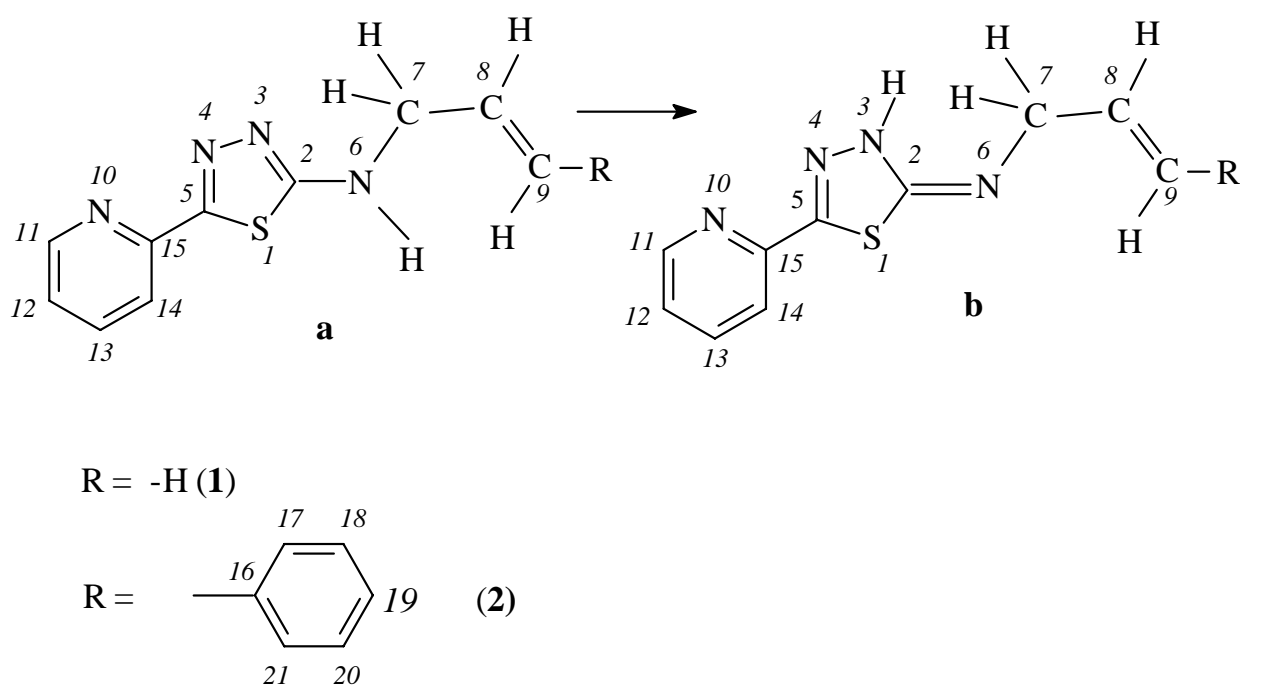

On account of the allyl- and pyridyl- substituents in the molecule, the exo- and endocyclic nitrogen atoms of 1,3,4-thiadiazole and pyridine rings may appear as amino-, pyrrole- or pyridinetype nitrogens. Consequently, allyl- (1) and 3-phenylallyl-(5-pyridin-2-yl-[1,3,4]thiadiazol-2-yl)amine (2) can exist as a a', b b', c', $\mathbf{a}_{\mathbf{0}} \mathbf{a}_{\mathbf{0}}$, tautomers (Figures 1-3).

Figure 2. Tautomers a', b' and c' of allyl- (1) and 3-phenylallyl-(5-pyridine-2-yl-[1,3,4]thiadiazol-2-yl)-amine (2), with atom numbering.

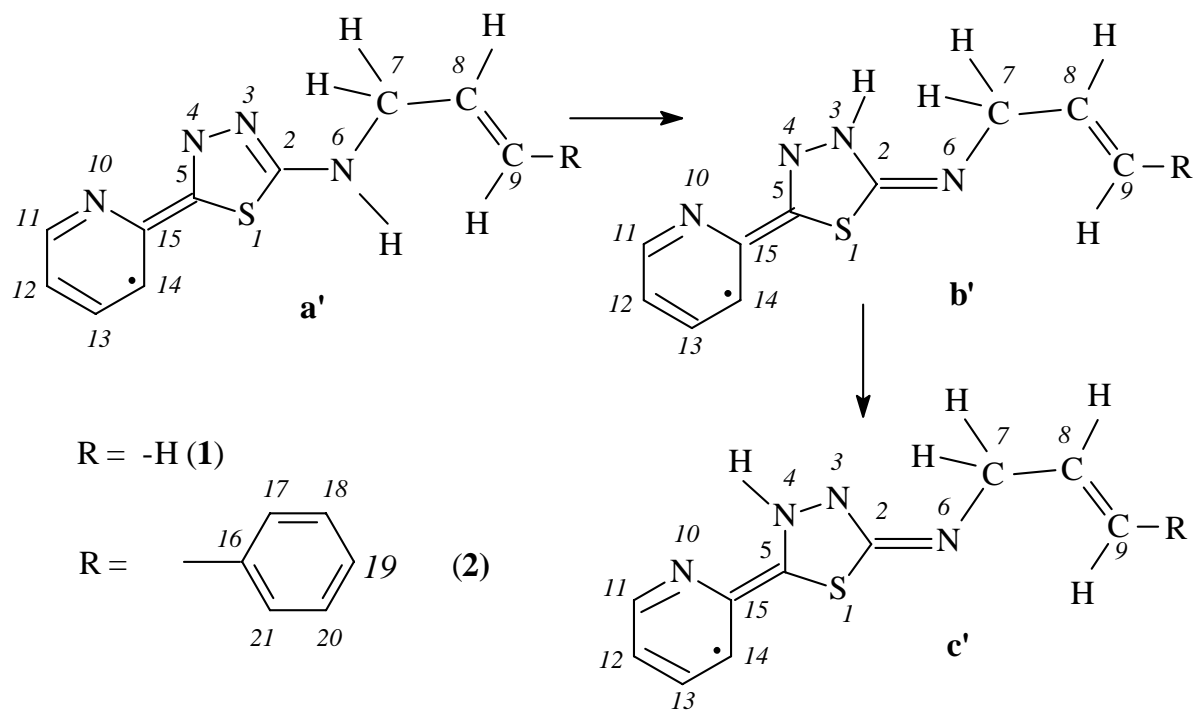


Previous $100 \mathrm{MHz}{ }^{1} \mathrm{H}-\mathrm{NMR}$ investigations of 2-amino-1,3,4-thiadiazole derivatives have shown the tautomeric equilibrium in solution between allyl- and 3-phenylallyl-(5-pyridin-2-yl[1,3,4]thiadiazol-2-yl)-amine (1a 2a， 1a' 2a')，3H-(allyl-(3-phenylallyl)-(5-pyridin-2-yl-[1,3,4]thiadiazol-2-ylidene)-amine (1b 2b, 1b’ 2b') and 4H-(allyl-(3-phenylallyl)-(5-pyridin-2-yl-[1,3,4]thiadiazol-2-ylidene)-amine (1c' 20') (Figures 1, 2) [6-9]. The intensities of the signals prove the interconversion between these tautomeric forms. According to the XRD data only one tautomer (type a) is present in the crystals of both compounds $\mathbf{1}$ and $\mathbf{2}$ [10]. In the solid state the exo-amino form a is stabilized by different $\mathrm{H}$ bonds, and the differences in the total energy between tautomers $\mathbf{a}$ and $\mathbf{b}$ are equal to -35.6 and $-34.3 \mathrm{~kJ} / \mathrm{mol}$ for $\mathbf{1}$ and 2, respectively, according to DFT level of theory calculations [10].

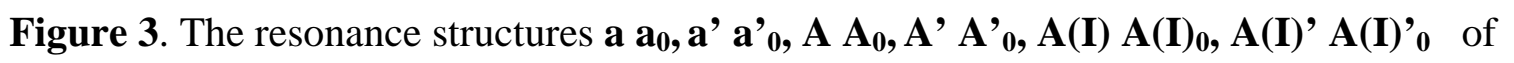
allyl-(1) and 3-phenylallyl-(5-pyridin-2-yl)-[1,3,4]thiadiazol-2-yl)-amine (2).

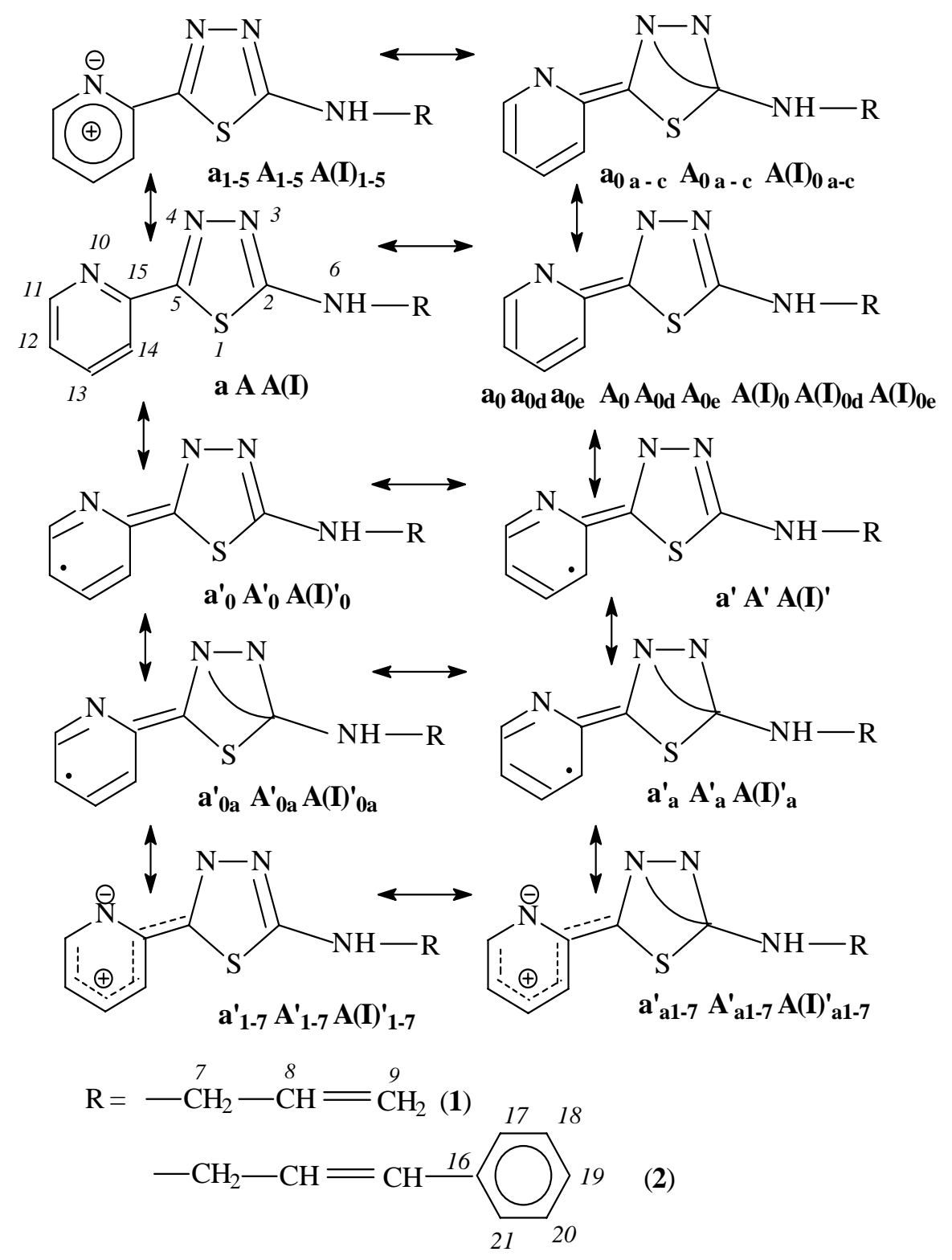


The calculated ${ }^{15} \mathrm{~N}$-, ${ }^{1} \mathrm{H}$ - chemical shifts and the ${ }^{15} \mathrm{~N}$-, ${ }^{1} \mathrm{H}$-, ${ }^{13} \mathrm{C}$ - signals of the respective ${ }^{15} \mathrm{~N}-,{ }^{1} \mathrm{H}$ and ${ }^{13} \mathrm{C}$-NMR spectra suggest changes in the electron configuration of the exo - and endocyclic nitrogen atoms of the 1,3,4-thiadiazole and pyridine rings. The aim of the present paper was to describe the electronic structure of the nitrogen atoms of tautomer 1a in the range of the chemical shifts of the $\mathrm{NH}$ group proton from $8.665 \mathrm{ppm}$ to $7.233 \mathrm{ppm}$.

Structural studies of 2-amino-[1,3,4]-thiadiazole derivatives have been performed in order to know the properties of the compounds with the marked bioactivity. The N6 and/or 5- substituted-2-amino[1,3,4]-thiadiazoles, depending on the nature of substituents, display varied pharmacological activities, and structural studies of new interesting [1,3,4]-thiadiazole derivatives with different medical applications may improve our understanding of their mode of action. Thus, they have shown potent activity against leukemia, melanoma and lung carcinoma. They are also known to be carbonic anhydrase inhibitors and some of them possess antimycobacterial, anesthetic, antidepressant and anxiolytic activity [11-21]. 2-Amino-[1,3,4]-thiadiazoles are also found in a new class of herbicides with a broad spectrum of activity [22], that act via inhibition of the enzyme imidazoleglycerol phosphate dehydrase. They are also useful as corrosion inhibitors [23].

\section{Results and Discussion}

The calculated chemical shifts of the ${ }^{15} \mathrm{~N}$ nitrogen atoms for type a and $\mathbf{b}$ tautomers of allyl-(1) and 3-phenylallyl-(5-pyridin-2-yl-[1,3,4]-thiadiazol-2-yl)-amine (2) occur in different ranges: from about 309 ppm to about -23 ppm for the type a tautomer and from about $-225 \mathrm{ppm}$ to about $-80 \mathrm{ppm}$ for the b one (Table 1, Figure 4) [10]. The amino N6 atom is strongly shielded in $\mathbf{1}$ (about -308 ppm) but in 2 the shielding decreases by a few ppm (to about -304 ppm). The shielding constants for the N3 and N10 atom in the 1,3,4-thiadiazole and pyridine rings, respectively, are almost equal, whereas the N4 atom is much less shielded [10].

Table 1. Calculated ${ }^{15} \mathrm{~N}$ - and ${ }^{1} \mathrm{H}-\mathrm{NMR}$ chemical shifts $\delta$ [ppm] of type $\mathbf{a}$ and $\mathbf{b}$ tautomers

\begin{tabular}{|c|c|c|c|}
\hline Compound & ${ }^{15} \mathbf{N}$ & \multicolumn{2}{|c|}{${ }^{1} \mathbf{H}$} \\
\hline $1 a 2 a$ & $-309--23$ & & \\
\hline 1a & N6 $\quad-131.57$ & H 14 & 8.125 \\
\hline & N3 $\quad-77.78$ & & \\
\hline $2 a$ & N10 -86.0 & H 6 & 7.5 \\
\hline & N6 $\quad-133.98$ & & \\
\hline 1b $2 b$ & $-225--80$ & & \\
\hline
\end{tabular}

In the ${ }^{1} \mathrm{H}-\mathrm{NMR}$ spectra the N6 nitrogen atom of $\mathbf{1 a}$ appears as an amine - type a, pyridine - type $\mathbf{A}$, pyrrole - type A (I) (Figures 1-3, 5, 6). The $5.81 \mathrm{ppm}$ value of the chemical shift for the proton of NH group of 1 recorded in $\mathrm{CDCl}_{3}$ solution at $500.16 \mathrm{MHz}$ [10] is in agreement with the resonances of the amino protons. The signal of the N6 nitrogen atom in the ${ }^{15} \mathrm{~N}-\mathrm{NMR}$ spectrum appears at $-308.58 \mathrm{ppm}$ [10] and supports the amino - type nitrogen. 
Figure 4. The linear regression of shielding constants $\sigma$ [ppm] versus chemical shifts $\delta$ [ppm] for $\mathbf{1 a}$ and $\mathbf{2 a .}$

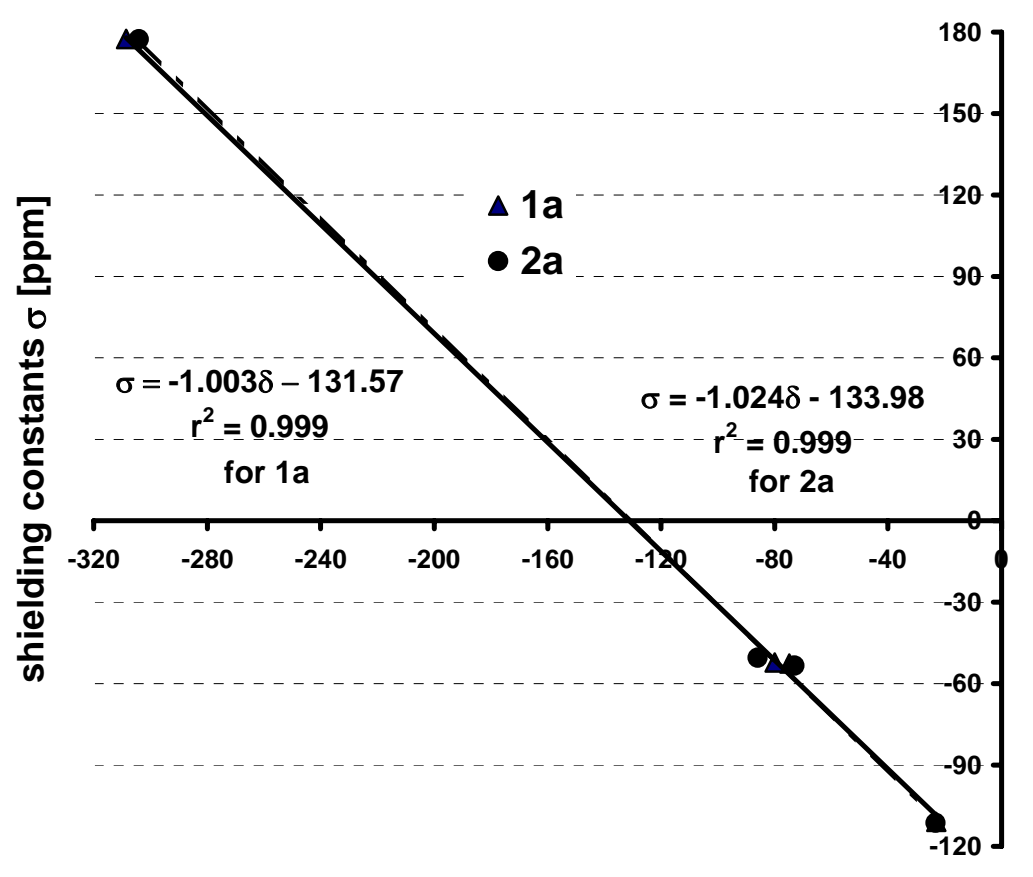

chemical shifts $\delta[p p m]$

Figure 5. The resonance structures $\mathbf{A} \mathbf{A}_{\mathbf{0}}$ of allyl-(1) and 3-phenylallyl-(5-pyridin-2-yl)[1,3,4]thiadiazol-2-yl)-amine (2)

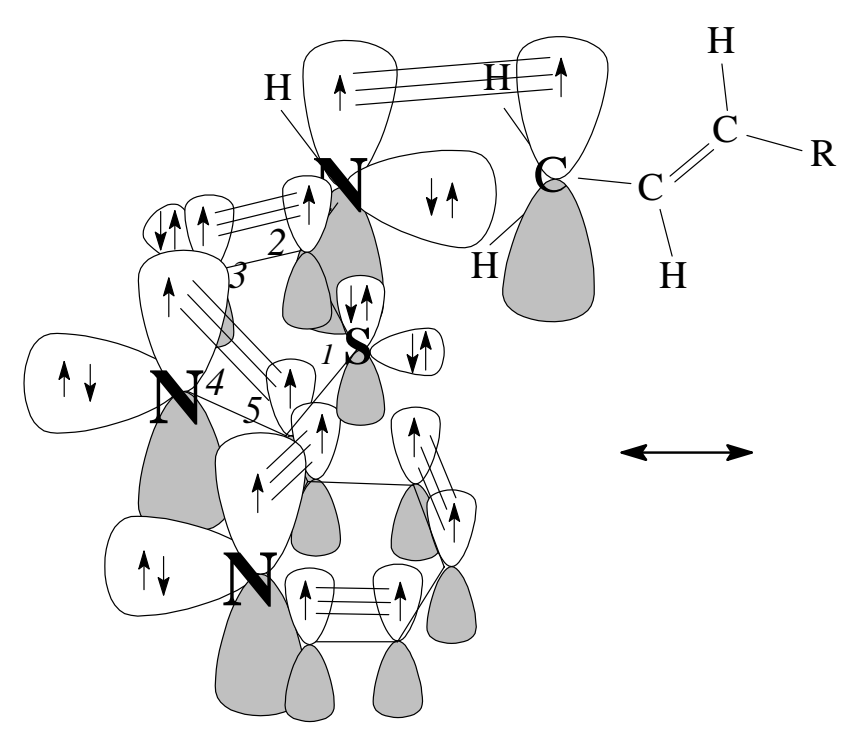

A

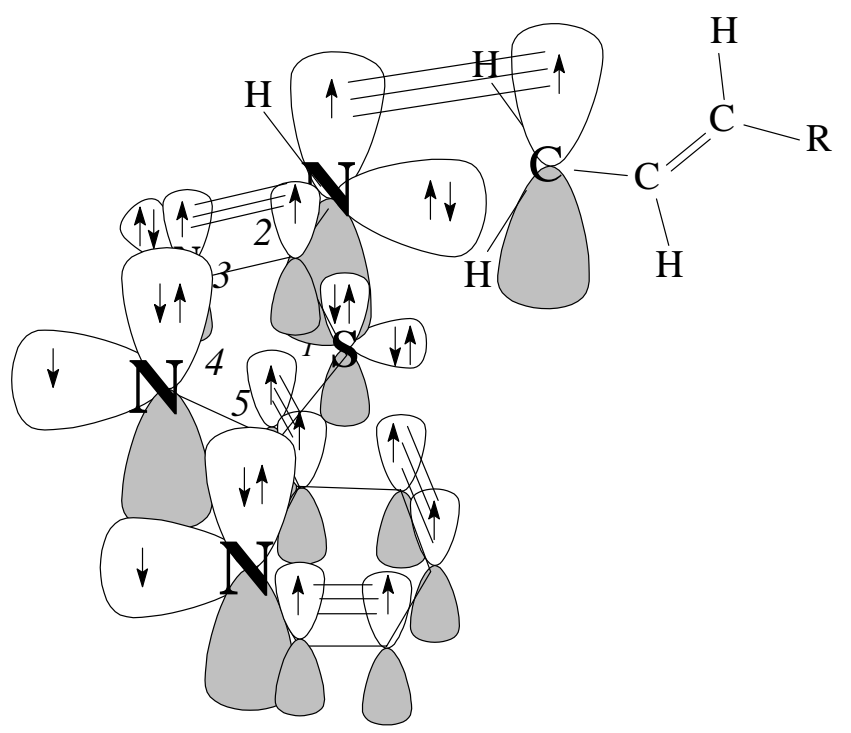

$\mathbf{A}_{\mathbf{0}}$ 
Figure 6. The resonance structures A(I) A(I) of allyl-(1) and 3-phenylallyl-(5-pyridin2-yl)-[1,3,4]thiadiazol-2-yl)-amine (2)

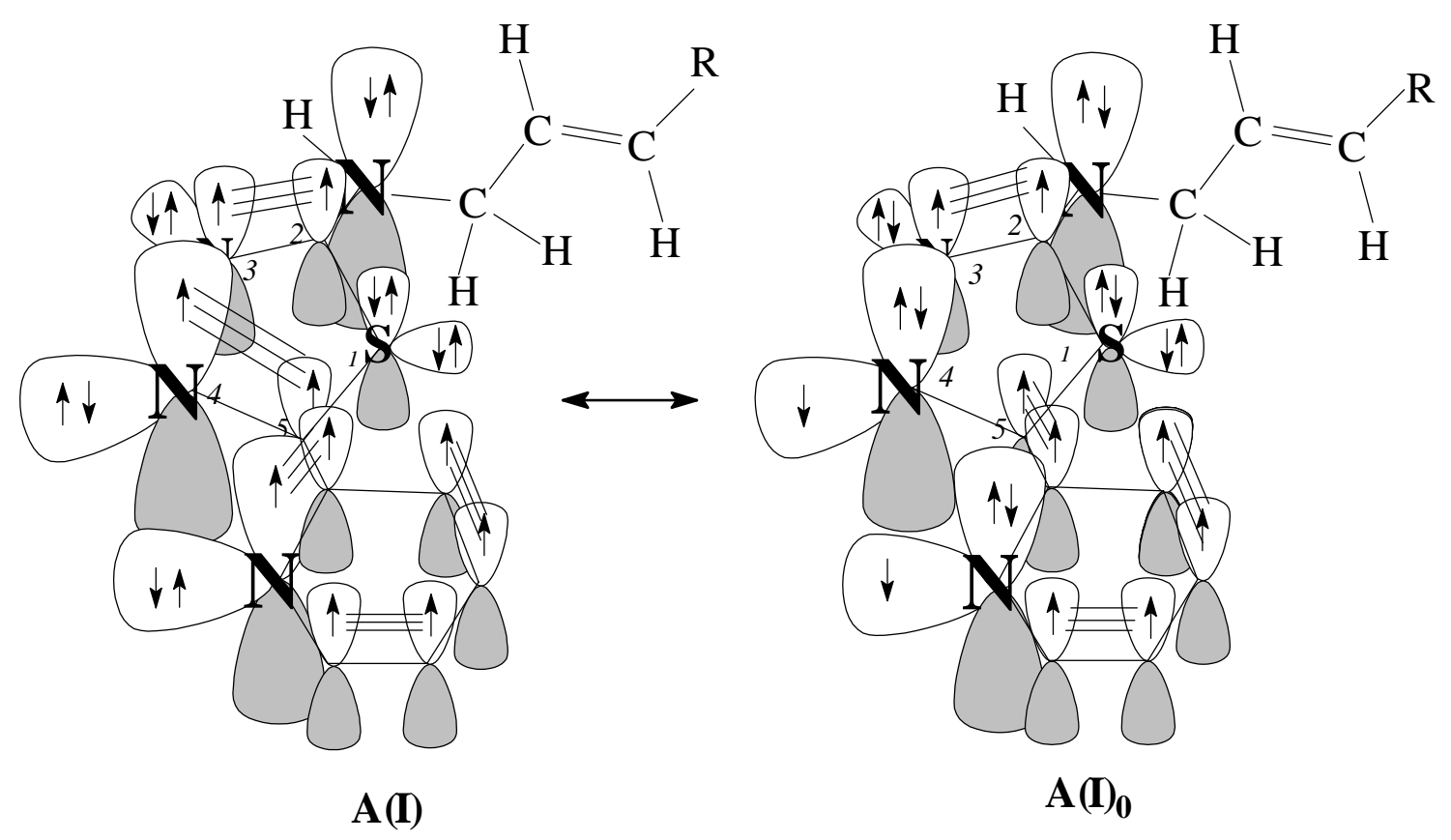

The differences in the coupling constants $J\left(\mathrm{H}_{8} \mathrm{H}_{9 \mathrm{~B}}\right) 17.6 \mathrm{~Hz}, J\left(\mathrm{H}_{8} \mathrm{H}_{7 \mathrm{C}}\right) 18.8 \mathrm{~Hz}, J\left(\mathrm{H}_{8} \mathrm{H}_{9 \mathrm{~A}}\right) 10.6 \mathrm{~Hz}$ $J\left(\mathrm{H}_{8} \mathrm{H}_{7 \mathrm{D}}\right) 11.2 \mathrm{~Hz}(100 \mathrm{MHz})$ [8] and the ${ }^{13} \mathrm{C}-\mathrm{NMR}$ signals of C9 allyl substituent at $117.99 \mathrm{ppm}$, C8 at $132.80 \mathrm{ppm}$ and $\mathrm{C} 7$ at $49.28 \mathrm{ppm}$ [10] support the negatively charged pyridine - type nitrogen atom and positively charged allyl cation. The coupling constants $J\left(\mathrm{H}_{8} \mathrm{H}_{9 \mathrm{~B}}\right) 17.6 \mathrm{~Hz}, J\left(\mathrm{H}_{8} \mathrm{H}_{9 \mathrm{~A}}\right) 10.6 \mathrm{~Hz}$, $J\left(\mathrm{H}_{8} \mathrm{H}_{9 \mathrm{~B}}\right) 17.3 \mathrm{~Hz}, J\left(\mathrm{H}_{8} \mathrm{H}_{9 \mathrm{~A}}\right) 10.9 \mathrm{~Hz}(100 \mathrm{MHz})$ [8] and $J\left(\mathrm{H}_{9 \mathrm{~B}} \mathrm{H}_{9 \mathrm{~A}}\right) 1.2 \mathrm{~Hz}(500 \mathrm{MHz})$ confirm the reversed electron demand of the 2p orbitals of the pyridine - type nitrogen and carbon atoms N6 C7 of 1. The exocyclic nitrogen atom, the pyridine - type, is occupied with eight electrons. The coupling constants $J\left(\mathrm{H}_{8} \mathrm{H}_{9 \mathrm{~B}}\right) 17.1 \mathrm{~Hz}, J\left(\mathrm{H}_{9 \mathrm{~B}} \mathrm{H}_{8}\right) 17.1 \mathrm{~Hz}, J\left(\mathrm{H}_{8} \mathrm{H}_{9 \mathrm{~A}}\right) 10.1 \mathrm{~Hz}, J\left(\mathrm{H}_{9 \mathrm{~A}} \mathrm{H}_{8}\right) 10.1 \mathrm{~Hz}, J\left(\mathrm{H}_{9 \mathrm{~B}} \mathrm{H}_{9 \mathrm{~A}}\right) 1.0$ $\mathrm{Hz}(500 \mathrm{MHz}$ ) [10] point to the lack of the differences in the spin states of electrons of 2p orbitals of pyridine - type nitrogen N6 and C7 atoms of 1, the exocyclic nitrogen atom N6 is surrounded by seven electrons. The calculated chemical shift of N6 at - $131.57 \mathrm{ppm}$ (Table 1) [10] supports pyridine - type nitrogen. The magnitude of the couplings $J\left(\mathrm{H}_{7} \mathrm{H}_{8}\right)=J\left(\mathrm{H}_{8} \mathrm{H}_{7}\right)=5.6 \mathrm{~Hz}(500 \mathrm{MHz})$ for 1 confirms a pyrrole - type nitrogen atom N6 $\mathbf{A ( I )}$ and the possible transformation of $\mathrm{sp}^{2} \Leftrightarrow \mathrm{sp}$.

The calculated signal of $\mathrm{H} 14$ at $8.125 \mathrm{ppm}$ (Table 1) as well as the ${ }^{1} \mathrm{H}-{ }^{1} \mathrm{H}$ coupling constants $J\left(\mathrm{H}_{12} \mathrm{H}_{14}\right) 1.0 \mathrm{~Hz}, J\left(\mathrm{H}_{11} \mathrm{H}_{14}\right) 0.5 \mathrm{~Hz}$ [10] of structure a confirm the absence of charges on the pyridine ring.

In the ${ }^{15} \mathrm{~N}-\mathrm{NMR}$ spectrum of 1a tautomer, the chemical shift of N10 at $-80.01 \mathrm{ppm}$ [10] supports the pyrrole - type nitrogen atom of the pyridyl substituent. The calculated chemical shift of N3 at $77.78 \mathrm{ppm}$ (Table 1) [10] confirm a pyridine - type nitrogen atom of tautomer 1a and the lack of the differences in the spin states of electrons of $2 \mathrm{p}$ orbitals of $\mathrm{N} 3 \mathrm{C} 2$. The ${ }^{1} \mathrm{H}-{ }^{13} \mathrm{C}$ HMQC correlation spectra show a correlation signal between $\mathrm{H} 14$ at $8.360 \mathrm{ppm}$ and $\mathrm{C} 15$ at $149.7 \mathrm{ppm}$. The above data prove the existence of the diradical resonance structures $\mathbf{a}_{0} \mathbf{A}_{\mathbf{0}} \mathbf{A}(\mathbf{I})_{\mathbf{0}} \mathbf{a}_{\mathbf{0}} \mathbf{A}^{\prime} \mathbf{y}_{\mathbf{0}} \mathbf{A}(\mathbf{I}){ }^{\prime} \mathbf{0}_{0}$ (Figures 3, 5 - 7) and the lack of the charges over the pyridine and 1,3,4 thiadiazole rings. 
Figure 7. The resonance structures of the pyridyl substituent

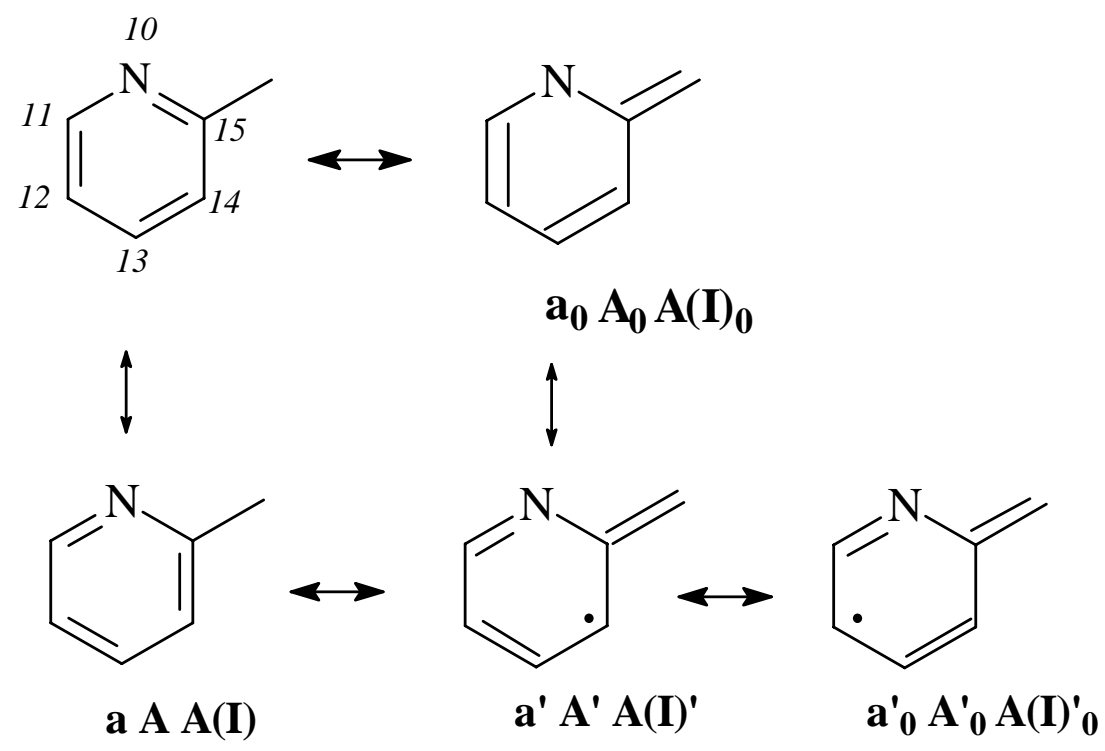

In the $2 \mathrm{D}{ }^{1} \mathrm{H}-{ }^{13} \mathrm{C}$ HMBC spectra the cross - peak between $\mathrm{H} 14$ and $\mathrm{C} 14$ at $8.150 \mathrm{ppm}$ and 119.7 ppm supports structures a A A(I) (Figures 3, 5 - 7). The pyridyl H14 proton of the diradical resonance structures $\mathbf{a}_{\mathbf{0}} \mathbf{A}_{\mathbf{0}} \mathbf{A}(\mathbf{I})_{0} \mathbf{a}_{\mathbf{0}} \mathbf{A}^{\mathbf{x}_{\mathbf{0}}} \mathbf{A}(\mathbf{I})^{\prime} \mathbf{0}_{0}$ is more intensly deshielded by about $0.2 \mathrm{ppm}$ in relation to the structure a $\mathbf{A} \mathbf{A}(\mathbf{I})$. The spectroscopic data support the conjugation of the aromatic $\pi$ electrons of the pyridyl substituent with the $\pi$ electrons of the $C=N$ double bond of the $1,3,4$ thiadiazole ring in solution.

The signals of the NH group protons and the pyridyl substituent in the ${ }^{1} \mathrm{H}-\mathrm{NMR}$ spectra $(100 \mathrm{MHz})$ support the a A and diradical resonance structures a' A', $\mathbf{a}_{\mathbf{0}} \mathbf{A}_{\mathbf{0}}$ (Figures 1-3, 5, 7). In the ${ }^{1} \mathrm{H}-\mathrm{NMR}$ spectra $1_{3} 1_{4}$ (Tables 2, 4) the H14 signals at 8.245 ppm - 8.145 ppm and 8.237 ppm - 8.137 ppm

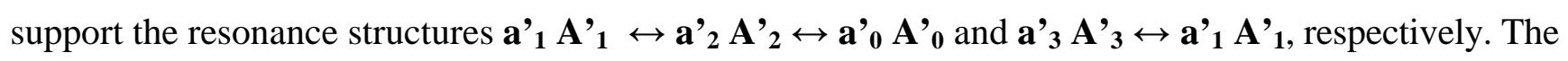
H14 signals at $8.242 \mathrm{ppm}-8.152 \mathrm{ppm}$ and $8.237 \mathrm{ppm}-8.148 \mathrm{ppm}$ (spectra 4, 2, 3, Tables 3, 4)

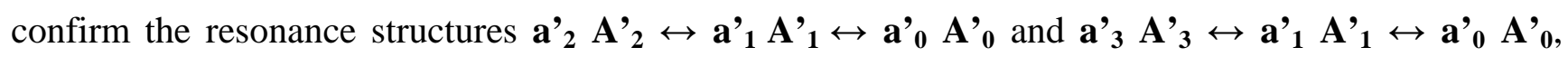
respectively. The $\mathrm{H} 14$ signals at $8.232 \mathrm{ppm}-8.143 \mathrm{ppm}$ and $8.223 \mathrm{ppm}-8.143 \mathrm{ppm}$ (spectra 1, 5,

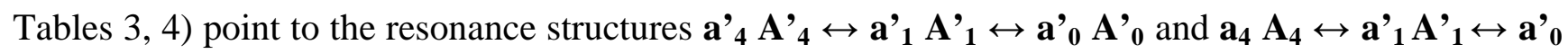
$\mathbf{A}_{\mathbf{0}}$, respectively. The H14 signals at $8.228 \mathrm{ppm}-8.138 \mathrm{ppm}$ (spectrum 6, Tables 3, 4) indicate the resonance structures $\mathbf{a}_{\mathbf{2}} \mathbf{A}_{\mathbf{2}} \leftrightarrow \mathbf{\mathbf { a } _ { 4 }} \mathbf{A}_{\mathbf{4}} \leftrightarrow \mathbf{a}_{\mathbf{1}} \mathbf{A}^{\prime}{ }_{1}$.

The H14 signals at $8.174 \mathrm{ppm}-8.023 \mathrm{ppm}, 8.174 \mathrm{ppm}-8.010 \mathrm{ppm}$ and $8.135 \mathrm{ppm}-7.998 \mathrm{ppm}$ (spectra $1_{8}, 1_{2}, 1_{1}$, Tables 2, 4) support the resonance structures $\mathbf{a}_{\mathbf{4}} \mathbf{A}_{\mathbf{4}} \leftrightarrow \mathbf{a}_{5} \mathbf{A}_{\mathbf{5}}, \mathbf{a}_{\mathbf{4}} \mathbf{A}_{\mathbf{4}} \leftrightarrow \mathbf{a}_{\mathbf{6}} \mathbf{A}_{\mathbf{6}}$ and $\mathbf{a}_{5} \mathbf{A}^{\mathbf{\prime}_{5}} \leftrightarrow \mathbf{a}_{\mathbf{6}} \mathbf{A}^{\prime}{ }_{6} \leftrightarrow \mathbf{a}_{7} \mathbf{A}^{\prime}{ }_{7}$, respectively. The H14 signals at $8.077 \mathrm{ppm}-7.974 \mathrm{ppm}$ (spectrum

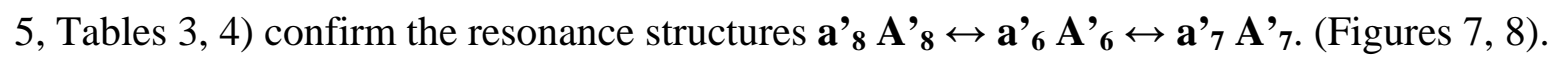


Figure 8. The resonance structures of the pyridyl substituent

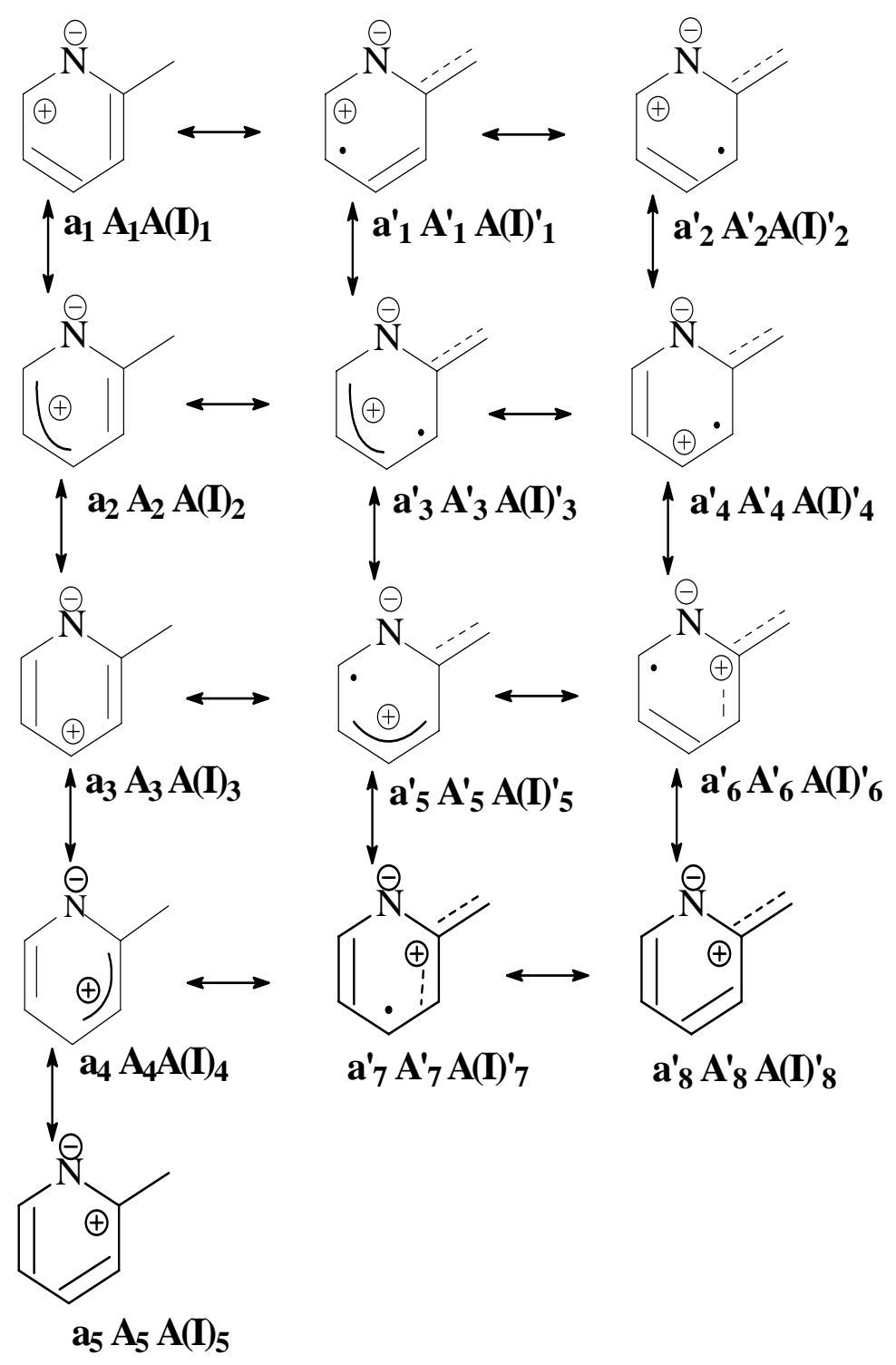

The H13 signals at $7.967 \mathrm{ppm}-7.869 \mathrm{ppm}, 7.954 \mathrm{ppm}-7.859 \mathrm{ppm}$ and $7.935 \mathrm{ppm}-7.837 \mathrm{ppm}$ (spectra $1_{8} 1_{2} 1_{1}$, Tables 2, 5) support the resonance structures $\mathbf{a}_{3} \mathbf{A}_{\mathbf{3}} \leftrightarrow \mathbf{a}_{\mathbf{3}} \mathbf{A}_{\mathbf{3}_{3}} \leftrightarrow \mathbf{a} \mathbf{A}, \mathbf{a}_{\mathbf{3}} \mathbf{A}^{\prime} \mathbf{3}_{\mathbf{3}} \leftrightarrow \mathbf{a}_{\mathbf{5}}$ $\mathbf{A}_{\mathbf{5}} \leftrightarrow \mathbf{a} \mathbf{A}$ and $\mathbf{a}_{\mathbf{4}_{\mathbf{4}}} \mathbf{A}^{\prime} \mathbf{4}_{\mathbf{4}} \leftrightarrow \mathbf{a}_{\mathbf{5}} \mathbf{A}^{\mathbf{\prime}_{5}} \leftrightarrow \mathbf{a} \mathbf{A}$, respectively. The H13 signals at $7.859 \mathrm{ppm}-7.688 \mathrm{ppm}$, $7.854 \mathrm{ppm}-7.681 \mathrm{ppm}$ (spectra $1_{3} 1_{4}$, Tables 2, 5) and $7.852 \mathrm{ppm}-7.683 \mathrm{ppm}$ ( spectrum 4, Tables 3, 5) point to the resonance structures $\mathbf{a}_{\mathbf{5}} \mathbf{A}_{\mathbf{5}_{5}} \leftrightarrow \mathbf{a}_{\mathbf{3}} \mathbf{A}_{\mathbf{3}} \leftrightarrow \mathbf{a}_{\mathbf{0}} \mathbf{A}_{\mathbf{0}}, \mathbf{a}_{\mathbf{3}} \mathbf{A}_{\mathbf{3}} \leftrightarrow \mathbf{a}_{\mathbf{5}} \mathbf{A}_{\mathbf{5}}$ and $\mathbf{a}_{\mathbf{3}} \mathbf{A}_{\mathbf{3}_{\mathbf{3}}} \leftrightarrow \mathbf{a}_{\mathbf{4}}$ $\mathbf{A}_{\mathbf{4}} \leftrightarrow \mathbf{a}_{\mathbf{0}} \mathbf{A}_{\mathbf{0}}$, respectively. The $\mathrm{H} 13$ signals at $7.852 \mathrm{ppm}-7.678 \mathrm{ppm}, 7.847 \mathrm{ppm}-7.674 \mathrm{ppm}$ (spectra 6, $1-3$, Tables 3, 5) and $7.838 \mathrm{ppm}-7.646 \mathrm{ppm}$, (spectrum 5, Tables 3, 5) confirm the resonance structures $\mathbf{a}_{\mathbf{3}} \mathbf{A}_{\mathbf{3}} \leftrightarrow \mathbf{a}_{\mathbf{4}} \mathbf{A}_{\mathbf{4}}, \mathbf{a}_{\mathbf{3}} \mathbf{A}_{\mathbf{3}} \leftrightarrow \mathbf{a}_{\mathbf{5}} \mathbf{A}_{\mathbf{5}} \leftrightarrow \mathbf{a}_{\mathbf{4}} \mathbf{A}_{\mathbf{4}}$ and $\mathbf{a}_{\mathbf{5}} \mathbf{A}_{\mathbf{5}} \leftrightarrow \mathbf{a}_{\mathbf{4}} \mathbf{A}_{\mathbf{4}}$, respectively. 
Table 2. The ${ }^{1} \mathrm{H}-\mathrm{NMR}$ chemical shifts $\delta$ [ppm] from TMS of $\mathbf{1}$.

\begin{tabular}{|c|c|c|c|c|}
\hline $\begin{array}{l}\text { Spectrum } \\
\text { No } \\
\text { (Solvent) }\end{array}$ & H 7 & H 8 & H 9 & Pyridin-2-yl \\
\hline $\begin{array}{l}1_{1} \\
\text { (DMSO) }\end{array}$ & $\begin{array}{c}3.922-4.061 \\
2 \mathrm{H} \mathrm{m}\end{array}$ & $\begin{array}{c}5.772-6.148 \\
1 \mathrm{H} \mathrm{m}\end{array}$ & $\begin{array}{c}5.104-5.399 \\
2 \mathrm{H} \mathrm{m}\end{array}$ & $\begin{array}{lll}8.637-8.562 & 1 \mathrm{H} & \mathrm{H} 11 \\
8.135-7.988 & 1 \mathrm{H} & \text { H13 H14 } \\
7.935-7.837 & 1 \mathrm{H} & \text { H12 H13 } \\
7.503-7.336 & 1 \mathrm{H} & \mathrm{H} 14 \text { H12 }\end{array}$ \\
\hline $\begin{array}{l}1_{2} \\
\text { (DMSO) }\end{array}$ & $\begin{array}{c}3.988-4.086 \\
2 \mathrm{H} \mathrm{m}\end{array}$ & $\begin{array}{c}5.809-6.187 \\
1 \mathrm{H} \mathrm{m}\end{array}$ & $\begin{array}{c}5.133-5.435 \\
2 \mathrm{H} \mathrm{m}\end{array}$ & $\begin{array}{lll}8.665-8.589 & 1 \mathrm{H} & \mathrm{H} 11 \\
8.174-8.010 & 1 \mathrm{H} & \mathrm{H} 13 \mathrm{H} 14 \\
7.954-7.859 & 1 \mathrm{H} & \mathrm{H} 12 \mathrm{H} 13 \\
7.517-7.381 & 1 \mathrm{H} & \mathrm{H} 14 \mathrm{H} 12\end{array}$ \\
\hline $\begin{array}{l}1_{3} \\
\left(\mathrm{CDCl}_{3}\right)\end{array}$ & $\begin{array}{c}4.003-4.086 \\
2 \mathrm{H} \mathrm{m}\end{array}$ & $\begin{array}{c}5.782-6.160 \\
1 \mathrm{H} \mathrm{m}\end{array}$ & $\begin{array}{c}5.191-5.482 \\
2 \mathrm{H} \mathrm{m}\end{array}$ & $\begin{array}{lll}8.606-8.530 & 1 \mathrm{H} & \mathrm{H} 11 \\
8.245-8.145 & 1 \mathrm{H} & \mathrm{H} 13 \mathrm{H} 14 \\
7.859-7.688 & 1 \mathrm{H} & \mathrm{H} 12 \mathrm{H} 13 \\
7.349-7.212 & 1 \mathrm{H} & \text { H14 H12 }\end{array}$ \\
\hline $\begin{array}{l}1_{4} \\
\left(\mathrm{CDCl}_{3}\right)\end{array}$ & $\begin{array}{c}4.003-4.086 \\
2 \mathrm{H} \mathrm{m}\end{array}$ & $\begin{array}{c}5.782-6.160 \\
1 \mathrm{H} \mathrm{m}\end{array}$ & $\begin{array}{c}5.191-5.482 \\
2 \mathrm{H} \mathrm{m}\end{array}$ & $\begin{array}{lll}8.601-8.525 & 1 \mathrm{H} & \mathrm{H} 11 \\
8.237-8.137 & 1 \mathrm{H} & \mathrm{H} 13 \mathrm{H} 14 \\
7.854-7.681 & 1 \mathrm{H} & \mathrm{H} 12 \mathrm{H} 13 \\
7.342-7.205 & 1 \mathrm{H} & \mathrm{H} 14 \mathrm{H} 12\end{array}$ \\
\hline $\begin{array}{l}1_{8}(\mathrm{DMSO} \\
\left.-\mathrm{D}_{2} \mathrm{O}\right)\end{array}$ & $\begin{array}{c}4.069-3.988 \\
2.5 \mathrm{H} \mathrm{m}\end{array}$ & $\begin{array}{c}5.804-6.180 \\
1.14 \mathrm{H} \mathrm{m}\end{array}$ & $\begin{array}{c}5.143-5.431 \\
2.21 \mathrm{H} \mathrm{m}\end{array}$ & $\begin{array}{lll}8.662-8.586 & 1.07 \mathrm{H} & \mathrm{H} 11 \\
8.174-8.023 & 1 \mathrm{H} & \mathrm{H} 13 \mathrm{H} 14 \\
7.967-7.869 & 1.42 \mathrm{H} & \mathrm{H} 12 \mathrm{H} 13 \\
7.532-7.395 & 1.21 \mathrm{H} & \mathrm{H} 14 \mathrm{H} 12 \\
\end{array}$ \\
\hline
\end{tabular}

The H12 signals at $7.532 \mathrm{ppm}-7.395 \mathrm{ppm}, 7.517 \mathrm{ppm}-7.381 \mathrm{ppm}$ and $7.503 \mathrm{ppm}-7.336 \mathrm{ppm}$ (spectra $1_{8} 1_{2} 1_{1}$, Tables 2, 6) confirm the resonance structures $\mathbf{a}_{5} \mathbf{A}_{\mathbf{5}} \leftrightarrow \mathbf{a}_{\mathbf{4}} \mathbf{A}_{\mathbf{4}} \leftrightarrow \mathbf{a}_{6} \mathbf{A}_{\mathbf{6}} \leftrightarrow \mathbf{a}_{\mathbf{0}} \mathbf{A}_{\mathbf{0}}$,

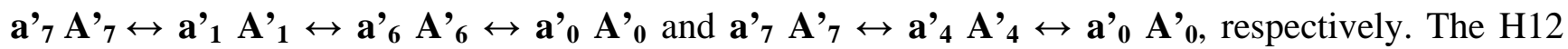
signals at $7.349 \mathrm{ppm}-7.212 \mathrm{ppm}$ and $7.342 \mathrm{ppm}-7.205 \mathrm{ppm}$ (spectra $1_{3} 1_{4}$, Tables 2, 6) support the resonance structures $\mathbf{a}_{\mathbf{4}} \mathbf{A}_{\mathbf{4}} \leftrightarrow \mathbf{a}_{\mathbf{2}} \mathbf{A}_{\mathbf{2}} \leftrightarrow \mathbf{a}_{\mathbf{1}} \mathbf{A}_{\mathbf{1}}$ and $\mathbf{a}_{\mathbf{4}} \mathbf{A}_{\mathbf{4}} \leftrightarrow \mathbf{a}_{\mathbf{1}} \mathbf{A}_{\mathbf{1}} \leftrightarrow \mathbf{a}_{\mathbf{0}} \mathbf{A}_{\mathbf{0}}$, respectively. The H12 signals at $7.397 \mathrm{ppm}-7.143 \mathrm{ppm}$ (spectrum 5, Tables 3, 6) support the resonance structures

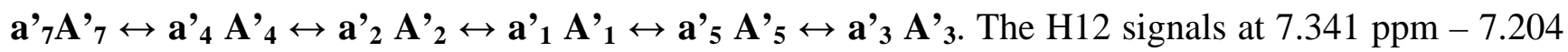
ppm, 7.336 ppm - 7.200 ppm and 7.331 ppm - 7.195 ppm (spectra 4, 1, 2, 6, 3, Tables 3, 6) support

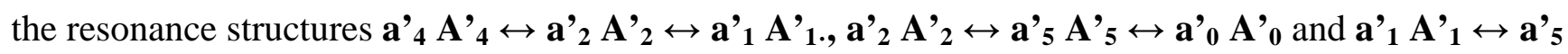
$\mathbf{A}_{5}$, respectively. 
Table 3. The ${ }^{1} \mathrm{H}-\mathrm{NMR}$ chemical shifts $\delta$ [ppm] from TMS of $\mathbf{1}$.

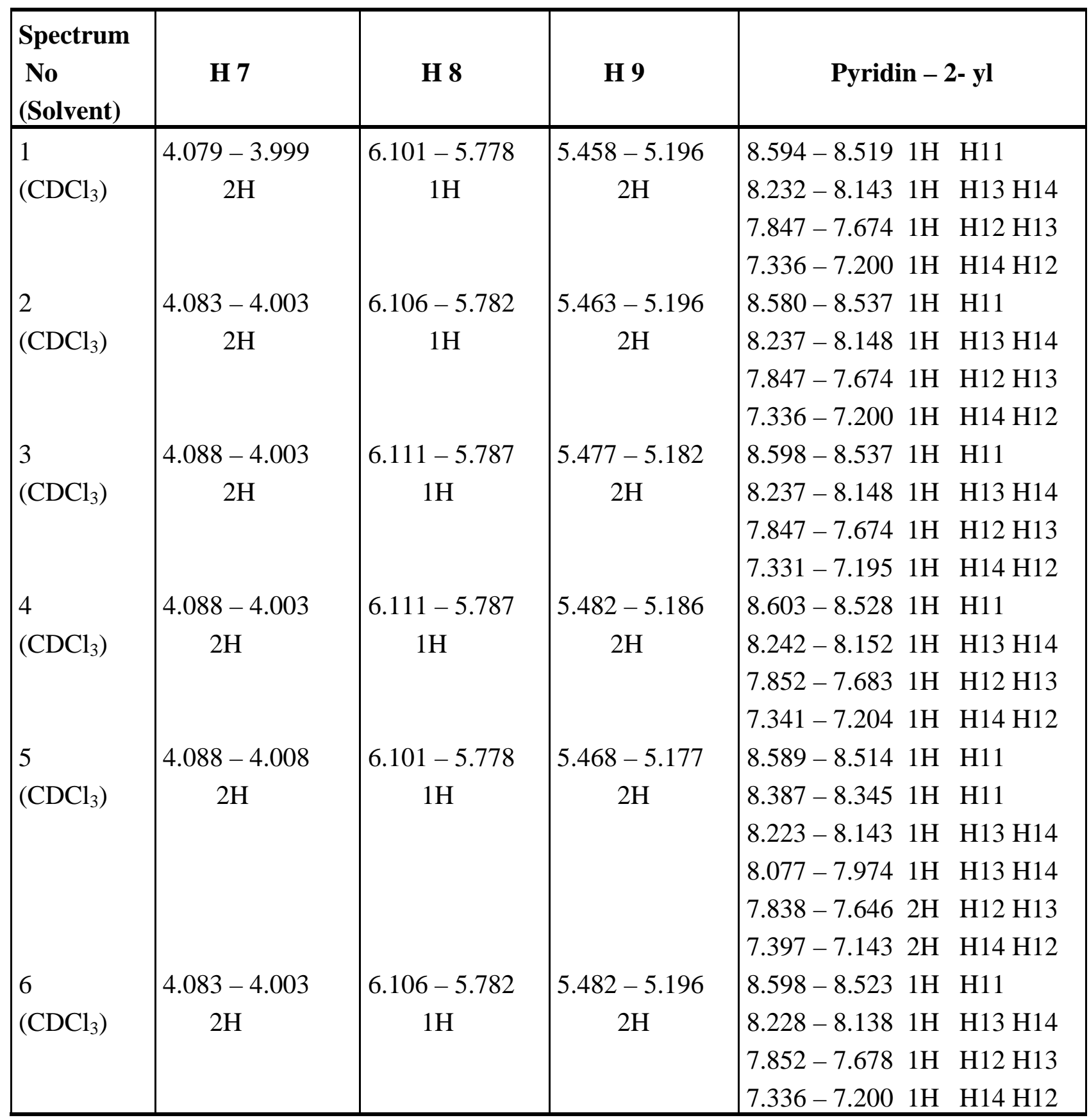

The signals of $\mathrm{H} 11$ at $8.665 \mathrm{ppm}-8.589 \mathrm{ppm}$ and $8.662 \mathrm{ppm}-8.586 \mathrm{ppm}$ (spectra $1_{2}, 1_{8}$, Tables 2, 7) point to the resonance structures $\mathbf{a}_{\mathbf{4}} \mathbf{A}_{\mathbf{4}} \leftrightarrow \mathbf{a}_{\mathbf{4}} \mathbf{A}_{\mathbf{4}} \leftrightarrow \mathbf{a}^{\prime} \mathbf{A}_{\mathbf{7}_{7}} \leftrightarrow \mathbf{a} \mathbf{A}$ and $\mathbf{a}_{\mathbf{4}} \mathbf{A}_{\mathbf{4}} \leftrightarrow \mathbf{a}_{\mathbf{6}} \mathbf{A}_{\mathbf{6}} \leftrightarrow \mathbf{a} \mathbf{A}$, respectively. The signals of $\mathrm{H} 11$ at $8.637 \mathrm{ppm}-8.562 \mathrm{ppm}$ (spectrum $1_{1}$, Tables 2, 7) support the

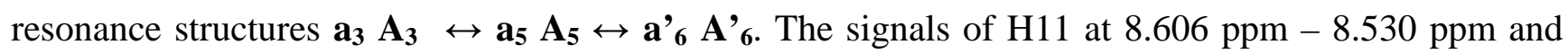
$8.601 \mathrm{ppm}-8.525 \mathrm{ppm}$ (spectra $1_{3}, 1_{4}$, Tables 2, 7) point to the resonance structures $\mathbf{a}_{\mathbf{7}} \mathbf{A}^{\mathbf{X}_{7}} \leftrightarrow \mathbf{a}_{\mathbf{1}} \mathbf{A}^{\mathbf{\prime}_{\mathbf{1}}}$

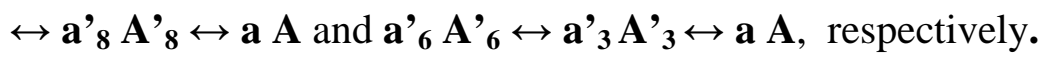

The signals of $\mathrm{H} 11$ at $8.603 \mathrm{ppm}-8.528 \mathrm{ppm}, 8.598 \mathrm{ppm}-8.537 \mathrm{ppm}$ and $8.598 \mathrm{ppm}-8.523$ ppm (spectra 4, 3, 6, Tables 3, 7) confirm the resonance structures $\mathbf{a}_{4} \mathbf{A}_{\mathbf{4}} \leftrightarrow \mathbf{a}_{5} \mathbf{A}_{\mathbf{5}} \leftrightarrow \mathbf{a} \mathbf{A}, \mathbf{a}_{5} \mathbf{A}_{\mathbf{5}}$ $\leftrightarrow \mathbf{a}_{\mathbf{8}} \mathbf{A}_{\mathbf{8}} \leftrightarrow \mathbf{a} \mathbf{A}$ and $\mathbf{a}_{\mathbf{5}} \mathbf{A}^{\prime}{ }_{5} \leftrightarrow \mathbf{a} \mathbf{A}^{\prime}$, respectively. The signals of H11 at $8.594 \mathrm{ppm}-8.519 \mathrm{ppm}$, $8.589 \mathrm{ppm}-8.514 \mathrm{ppm}$ and $8.580 \mathrm{ppm}-8.537 \mathrm{ppm}$ (spectra 1, 5, 2, Tables 3, 7) indicate the $\mathbf{a}_{\mathbf{5}} \mathbf{A}^{\mathbf{A}_{\mathbf{5}}}$ 


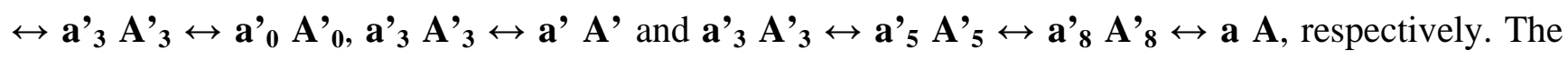
signals of $\mathrm{H} 11$ at $8.387 \mathrm{ppm}-8.345$ ppm (spectrum 5 , Tables 3 , 7) point to the resonance structures $\mathbf{a}_{1} \mathbf{A}^{\prime}{ }_{1} \leftrightarrow \mathbf{a}_{2} \mathbf{A}^{\prime}{ }_{2} \leftrightarrow \mathbf{a}_{1} \mathbf{A}_{1}$.

Table 4. The ${ }^{1} \mathrm{H}-\mathrm{NMR}$ chemical shifts $\delta$ [ppm] from TMS of $\mathbf{1}$.

\begin{tabular}{|c|c|c|c|}
\hline \multirow{2}{*}{$\begin{array}{l}\text { Spectrum No } \\
\text { (Solvent) }\end{array}$} & \multicolumn{3}{|c|}{ Pyridin - 2- yl } \\
\hline & H 14 - of the structures & H 14, H 13 & H 13 - of the structures \\
\hline $1_{3}\left(\mathrm{CDCl}_{3}\right)$ & $a^{\prime}{ }_{1} A^{\prime}{ }_{1} \leftrightarrow a^{\prime}{ }_{2} A^{\prime}{ }_{2} \leftrightarrow a^{\prime}{ }_{0} A^{\prime}{ }_{0}$ & $8.245-8.145$ & $\mathbf{a}^{\prime}{ }_{1} \mathbf{A}^{\prime}{ }_{1} \leftrightarrow \mathbf{a}_{2} \mathbf{A}^{\prime}{ }_{2}$ \\
\hline $1_{4}\left(\mathrm{CDCl}_{3}\right)$ & $\mathrm{a}^{\prime}{ }_{3} \mathbf{A}^{\prime}{ }_{3} \leftrightarrow \mathrm{a}^{\prime}{ }_{1} \mathbf{A}^{\prime}{ }_{1}$ & $8.237-8.137$ & $\mathbf{a}_{2} \mathbf{A}_{2} \leftrightarrow \mathbf{a}^{\prime}{ }_{3} \mathbf{A}^{\prime}{ }_{3}$ \\
\hline $4\left(\mathrm{CDCl}_{3}\right)$ & $a^{\prime}{ }_{2} A^{\prime}{ }_{2} \leftrightarrow a^{\prime} A^{\prime}{ }_{1} \leftrightarrow a^{\prime}{ }_{0} A^{\prime}{ }_{0}$ & $8.242-8.152$ & $\mathbf{a}_{1} \mathrm{~A}_{1} \leftrightarrow \mathbf{a}^{\prime}{ }_{1} \mathrm{~A}^{\prime}{ }_{1} \leftrightarrow \mathrm{a} A$ \\
\hline 2, $3\left(\mathrm{CDCl}_{3}\right)$ & $a^{\prime}{ }_{3} A^{\prime}{ }_{3} \leftrightarrow a^{\prime} A^{\prime}{ }_{1} \leftrightarrow a^{\prime} A^{\prime}{ }_{0}$ & $8.237-8.148$ & $\mathbf{a}_{2} \mathbf{A}_{2} \leftrightarrow \mathbf{a}^{\prime} \mathbf{A}^{\prime}$ \\
\hline $1\left(\mathrm{CDCl}_{3}\right)$ & $a^{\prime}{ }_{4} A^{\prime}{ }_{4} \leftrightarrow a^{\prime}{ }_{1} A^{\prime}{ }_{1} \leftrightarrow a^{\prime} A^{\prime}{ }^{\prime}{ }_{0}$ & $8.232-8.143$ & $\mathbf{a}^{\prime}{ }_{3} \mathbf{A}^{\prime}{ }_{3} \leftrightarrow \mathbf{a}^{\prime} \mathbf{A}^{\prime}$ \\
\hline $5\left(\mathrm{CDCl}_{3}\right)$ & $\mathbf{a}_{\mathbf{4}} \mathbf{A}_{\mathbf{4}} \leftrightarrow \mathbf{a}^{\prime}{ }_{1} \mathbf{A}^{\prime}{ }_{1} \leftrightarrow \mathbf{a}^{\prime} \mathbf{A}^{\prime}{ }_{0}$ & $8.223-8.143$ & $\mathbf{a}_{4} \mathbf{A}_{4} \leftrightarrow \mathbf{a}^{\prime}{ }_{3} \mathbf{A}^{\prime}{ }_{3} \leftrightarrow \mathbf{a}^{\prime} \mathbf{A}^{\prime}$ \\
\hline $6\left(\mathrm{CDCl}_{3}\right)$ & $\mathbf{a}_{2} \mathbf{A}_{2} \leftrightarrow \mathbf{a}_{4} \mathbf{A}_{4} \leftrightarrow \mathbf{a}^{\prime}{ }_{1} \mathbf{A}^{\prime}{ }_{1}$ & $8.228-8.138$ & $\mathbf{a}_{2} \mathbf{A}_{2} \leftrightarrow \mathbf{a}_{4} \mathbf{A}_{4} \leftrightarrow \mathbf{a}^{\prime} \mathbf{A}^{\prime}{ }_{3}$ \\
\hline $1_{8}\left(\mathrm{DMSO}-\mathrm{D}_{2} \mathrm{O}\right)$ & $\mathbf{a}^{\prime}{ }_{4} \mathbf{A}^{\prime}{ }_{4} \leftrightarrow \mathbf{a}^{\prime}{ }_{5} \mathbf{A}^{\prime}{ }_{5}$ & $8.174-8.023$ & $\mathbf{a}_{4} \mathbf{A}_{4} \leftrightarrow \mathbf{a}^{\prime}{ }_{3} \mathbf{A}^{\prime}{ }_{3}$ \\
\hline $1_{2}(\mathrm{DMSO})$ & $a^{\prime}{ }_{4} A^{\prime}{ }_{4} \leftrightarrow a^{\prime}{ }_{6} A^{\prime}{ }_{6}$ & $8.174-8.010$ & $\mathbf{a}_{4} \mathbf{A}_{4} \leftrightarrow \mathbf{a}^{\prime}{ }_{5} \mathbf{A}^{\prime}{ }_{5}$ \\
\hline $1_{1}(\mathrm{DMSO})$ & $\mathbf{a}_{5}{ }_{5} \mathbf{A}^{\prime}{ }_{5} \leftrightarrow \mathbf{a}_{6} \mathbf{A}^{\prime}{ }_{6} \leftrightarrow \mathbf{a}^{\prime}{ }_{7} \mathbf{A}^{\prime}{ }_{7}$ & $8.135-7.998$ & $a^{\prime}{ }_{5} A^{\prime}{ }_{5} \leftrightarrow a^{\prime}{ }_{3} A^{\prime}{ }_{3}$ \\
\hline $5\left(\mathrm{CDCl}_{3}\right)$ & $\mathbf{a}_{8}{ }_{8} \mathbf{A}^{\prime}{ }_{8} \leftrightarrow \mathbf{a}_{6} \mathbf{A}^{\prime}{ }_{6} \leftrightarrow \mathbf{a}_{7} \mathbf{A}^{\prime}{ }_{7}$ & $8.077-7.974$ & $a^{\prime}{ }_{3} A^{\prime}{ }_{3} \leftrightarrow a^{\prime}{ }_{5} A^{\prime}{ }_{5} \leftrightarrow a^{\prime}{ }_{4} A^{\prime}{ }_{4}$ \\
\hline
\end{tabular}

The signals of $\mathrm{H} 14$ at $7.532 \mathrm{ppm}$ - 7.395 ppm, 7.517 ppm - $7.381 \mathrm{ppm}$ and 7.503 ppm - 7.336 ppm (spectra $1_{8} 1_{2}, 1_{1}$, Tables 2, 6) confirm the resonance structures $\mathbf{a}_{\mathbf{1}} \mathbf{A}_{\mathbf{1}} \leftrightarrow \mathbf{a}_{\mathbf{1}} \mathbf{A}^{\prime} \mathbf{1}_{\mathbf{1}} \leftrightarrow \mathbf{a} \mathbf{A}, \mathbf{a}_{\mathbf{2}} \mathbf{A}_{\mathbf{2}} \leftrightarrow$ $\mathbf{a}_{3} \mathbf{A}^{\prime}{ }_{3} \leftrightarrow \mathbf{a} \mathbf{A}$ and $\mathbf{a}_{2} \mathbf{A}_{2} \leftrightarrow \mathbf{a}_{4} \mathbf{A}^{\prime}{ }_{4} \leftrightarrow \mathbf{a} \mathbf{A}$, respectively. The signals of H14 at $7.349 \mathrm{ppm}-7.212$ ppm and $7.342 \mathrm{ppm}-7.205 \mathrm{ppm}$ (spectra $1_{3} 1_{4}$, Tables 2, 6) support the resonance structures $\mathbf{a}_{3} \mathbf{A}^{\mathbf{\prime}_{3}}$ $\leftrightarrow \mathbf{a}^{\prime} \mathbf{A}^{\prime}$ and $\mathbf{a}_{4} \mathbf{A}^{\mathbf{A}_{4}} \leftrightarrow \mathbf{a}^{\prime} \mathbf{A}^{\prime}$, respectively. The signals of H14 at $7.397 \mathrm{ppm}-7.143 \mathrm{ppm}$ ( spectrum 5, Tables 3, 6) confirm the resonance structures $\mathbf{a}_{\mathbf{1}} \mathbf{A}_{\mathbf{1}} \leftrightarrow \mathbf{a}_{\mathbf{3}} \mathbf{A}_{\mathbf{3}} \leftrightarrow \mathbf{a}_{\mathbf{4}} \mathbf{A}_{\mathbf{4}} \leftrightarrow \mathbf{a}_{\mathbf{5}} \mathbf{A}_{\mathbf{5}} \leftrightarrow \mathbf{a}_{\mathbf{6}} \mathbf{A}_{\mathbf{6}} \leftrightarrow$ $\mathbf{a}_{7} \mathbf{A}^{\prime}{ }_{7}$. The signals of $\mathrm{H} 14$ at $7.341 \mathrm{ppm}-7.204 \mathrm{ppm}, 7.336 \mathrm{ppm}-7.200 \mathrm{ppm}$ and $7.331 \mathrm{ppm}-$ 7.195 ppm (spectra 4, 1, 2, 6, 3, Tables 3, 6) confirm the resonance structures $\mathbf{a}_{\mathbf{4}} \mathbf{A}^{\mathbf{\prime}_{\mathbf{4}}} \leftrightarrow \mathbf{a} \mathbf{A}, \mathbf{a}_{\mathbf{4}} \mathbf{A}^{\mathbf{\prime}_{4}}$ $\leftrightarrow$ a' A' $\leftrightarrow$ a' ${ }_{5} \mathbf{A}^{\prime}{ }_{5}$ and $\mathbf{a}_{5} \mathbf{A}^{\prime}{ }_{5} \leftrightarrow \mathbf{a}^{\prime} \mathbf{A}^{\prime} \leftrightarrow \mathbf{a}^{\prime} \mathbf{A}^{\prime} \mathbf{6}_{6}$, respectively.

The signals of $\mathrm{H} 13$ at $8.245 \mathrm{ppm}-8.145 \mathrm{ppm}$ and $8.237 \mathrm{ppm}-8.137 \mathrm{ppm}$ (spectra $1_{3}, 1_{4}$, Tables 2, 4) point to the $\mathbf{a}_{1} \mathbf{A}^{\prime}{ }_{1} \leftrightarrow \mathbf{a}_{\mathbf{2}} \mathbf{A}^{\prime}{ }_{2}$ and $\mathbf{a}_{\mathbf{2}} \mathbf{A}_{\mathbf{2}} \leftrightarrow \mathbf{a}_{3} \mathbf{A}^{\mathbf{\prime}_{3}}$ resonance structures, respectively. The signals of $\mathrm{H} 13$ at $8.242 \mathrm{ppm}$ - $8.152 \mathrm{ppm}, 8.237 \mathrm{ppm}-8.148 \mathrm{ppm}$ and $8.232 \mathrm{ppm}-8.143 \mathrm{ppm}$ (spectra 4, 2, 3, 1, Tables 3, 4 ) confirm the resonance structures $\mathbf{a}_{1} \mathbf{A}_{\mathbf{1}} \leftrightarrow \mathbf{a}_{1} \mathbf{A}^{\prime} \mathbf{1}_{\mathbf{1}} \leftrightarrow \mathbf{a} \mathbf{A}, \mathbf{a}_{\mathbf{2}} \mathbf{A}_{\mathbf{2}} \leftrightarrow \mathbf{a}$ $\mathbf{A}^{\prime}$ and $\mathbf{a}_{3} \mathbf{A}_{3} \leftrightarrow \mathbf{a}^{\prime} \mathbf{A}^{\prime}$, respectively.

The signals of $\mathrm{H} 13$ at $8.228 \mathrm{ppm}-8.138 \mathrm{ppm}$ and $8.223 \mathrm{ppm}-8.143 \mathrm{ppm}$ (spectra 6, 5, Tables 3, 4) confirm the resonance structures $\mathbf{a}_{2} \mathbf{A}_{2} \leftrightarrow \mathbf{a}_{4} \mathbf{A}_{4} \leftrightarrow \mathbf{a}_{3} \mathbf{A}_{\mathbf{3}_{3}}$ and $\mathbf{a}_{4} \mathbf{A}_{4} \leftrightarrow \mathbf{a}_{3} \mathbf{A}^{\prime}{ }_{3} \leftrightarrow \mathbf{a}^{\prime} \mathbf{A}^{\prime}$, respectively. The signals of $\mathrm{H} 13$ at $8.174 \mathrm{ppm}-8.023 \mathrm{ppm}, 8.174 \mathrm{ppm}-8.010 \mathrm{ppm}$ and $8.135 \mathrm{ppm}-$ $7.988 \mathrm{ppm}$ (spectra $1_{8}, 1_{2}, 1_{1}$, Tables 2, 4) support the resonance structures $\mathbf{a}_{\mathbf{4}} \mathbf{A}_{\mathbf{4}} \leftrightarrow \mathbf{a}_{3} \mathbf{A}^{\prime}{ }_{3}, \mathbf{a}_{\mathbf{4}} \mathbf{A}_{\mathbf{4}} \leftrightarrow$ $\mathbf{a}_{5} \mathbf{A}_{5}$ and $\mathbf{a}_{5} \mathbf{A}_{5} \leftrightarrow \mathbf{a}_{3} \mathbf{A}_{3}$, respectively. The signals of $\mathrm{H13}$ at $8.077 \mathrm{ppm}-7.974 \mathrm{ppm}$ (spectrum 5,

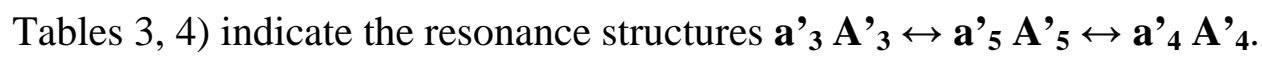


Table 5. The ${ }^{1} \mathrm{H}-\mathrm{NMR}$ chemical shifts $\delta$ [ppm] from TMS of $\mathbf{1}$.

\begin{tabular}{|c|c|c|c|}
\hline \multirow{2}{*}{$\begin{array}{l}\text { Spectrum No } \\
\text { (Solvent) }\end{array}$} & \multicolumn{3}{|c|}{ Pyridin - 2- yl } \\
\hline & H 13 - of the structures & H 13, H 12 & H 12 - of the structures \\
\hline $1_{8}\left(\mathrm{DMSO}-\mathrm{D}_{2} \mathrm{O}\right)$ & $\mathbf{a}_{3} \mathbf{A}_{3} \leftrightarrow \mathbf{a}_{3}{ }_{3} \mathbf{A}^{\prime}{ }_{3} \leftrightarrow \mathbf{a A}$ & $7.967-7.869$ & $\mathbf{a}_{5} \mathbf{A}_{5} \leftrightarrow \mathbf{a}_{1} \mathbf{A}_{1} \leftrightarrow \mathbf{a}^{\prime}{ }_{8} \mathbf{A}^{\prime}{ }_{8} \leftrightarrow \mathbf{a} A$ \\
\hline $1_{2}(\mathrm{DMSO})$ & $\mathbf{a}_{3}{ }_{3} \mathbf{\prime}_{3} \leftrightarrow \mathbf{a}_{5} \mathbf{A}^{\prime}{ }_{5} \leftrightarrow \mathrm{aA}$ & $7.954-7.859$ & $\mathbf{a}_{8} \mathbf{A}^{\prime}{ }_{8} \leftrightarrow \mathbf{a}_{7} \mathbf{A}^{\prime}{ }_{7}$ \\
\hline $1_{1}(\mathrm{DMSO})$ & $\mathbf{a}^{\prime}{ }_{4} \mathbf{A}^{\prime} \leftrightarrow \mathbf{a}_{5}{ }^{\prime} \mathbf{A}_{5}{ }_{5} \mathrm{aA}$ & $7.935-7.837$ & $\mathbf{a}_{7} \mathbf{A}^{\prime}{ }_{7} \leftrightarrow \mathbf{a}_{6}{ } \mathbf{A}^{\prime}{ }_{6}$ \\
\hline $1_{3}\left(\mathrm{CDCl}_{3}\right)$ & $\mathbf{a}_{5} \mathbf{A}^{\prime}{ }_{5} \leftrightarrow \mathbf{a}_{3} \mathbf{A}^{\prime}{ }_{3} \leftrightarrow \mathbf{a}_{0} \mathbf{A}^{\prime}{ }_{0}$ & $7.859-7.688$ & $\mathbf{a}_{7} \mathbf{A}^{\prime}{ }_{7} \leftrightarrow \mathbf{a}^{\prime} \mathbf{A}^{\prime}{ }_{1} \leftrightarrow \mathbf{a A}$ \\
\hline $1_{4}\left(\mathrm{CDCl}_{3}\right)$ & $\mathbf{a}_{3} \mathbf{A}^{\prime}{ }_{3} \leftrightarrow \mathbf{a}_{5} \mathbf{A}^{\prime}{ }_{5}$ & $7.854-7.681$ & $\mathbf{a}_{1} \mathbf{A}_{1} \leftrightarrow \mathbf{a}_{2}{ }_{2} \mathbf{A}^{\prime}{ }_{2} \leftrightarrow \mathbf{a}^{\prime} \mathbf{A}^{\prime}$ \\
\hline $4\left(\mathrm{CDCl}_{3}\right)$ & $a^{\prime}{ }_{3} A^{\prime}{ }_{3} \leftrightarrow a^{\prime}{ }_{4}{ }^{\prime}{ }_{4} \leftrightarrow a_{0} A^{\prime}{ }_{0}$ & $7.852-7.683$ & $\mathbf{a}_{2} \mathbf{A}_{2} \leftrightarrow \mathbf{a}_{2}{ }^{\prime} \mathbf{A}^{\prime}{ }_{2} \leftrightarrow \mathbf{a A}$ \\
\hline $6\left(\mathrm{CDCl}_{3}\right)$ & $\mathbf{a}^{\prime} \mathrm{A}^{\prime}{ }_{3} \leftrightarrow \mathbf{a}_{4} \mathrm{~A}^{\prime}{ }_{4}$ & $7.852-7.678$ & $\mathbf{a}_{2} \mathbf{A}_{2} \leftrightarrow \mathbf{a}^{\prime}{ }_{1} \mathbf{A}^{\prime}{ }_{1}$ \\
\hline $1-3\left(\mathrm{CDCl}_{3}\right)$ & $\mathbf{a}_{3}{ }^{\prime} \mathbf{A}^{\prime}{ }_{3} \leftrightarrow \mathbf{a}_{5} \mathbf{A}^{\prime}{ }_{5} \leftrightarrow \mathbf{a}^{\prime}{ }_{4} \mathbf{A}^{\prime}{ }_{4}$ & $7.847-7.674$ & $\mathbf{a}^{\prime}{ }_{1} \mathbf{A}^{\prime}{ }_{1} \leftrightarrow \mathbf{a}^{\prime}{ } \mathbf{A}^{\prime}{ }_{2}$ \\
\hline $5\left(\mathrm{CDCl}_{3}\right)$ & $\mathbf{a}^{\prime} \mathbf{A}^{\prime}{ }_{5} \leftrightarrow \mathbf{a}^{\prime}{ }_{4} \mathbf{A}^{\prime}{ }_{4}$ & $7.838-7.646$ & $\mathbf{a}^{\prime}{ }_{6} \mathbf{A}^{\prime}{ }_{6}^{\prime} \leftrightarrow \mathbf{a}^{\prime} \mathbf{A}^{\prime}{ }_{1}^{\prime} \leftrightarrow \mathbf{a}_{3} \mathbf{A}^{\prime}{ }_{3}$ \\
\hline
\end{tabular}

Table 6. The ${ }^{1} \mathrm{H}-\mathrm{NMR}$ chemical shifts $\delta$ [ppm] from TMS of $\mathbf{1}$.

\begin{tabular}{|c|c|c|c|}
\hline \multirow{2}{*}{$\begin{array}{l}\text { Spectrum No } \\
\text { (Solvent) }\end{array}$} & \multicolumn{3}{|c|}{ Pyridin - 2- yl } \\
\hline & H 12 - of the structures & H 12, H 14 & H 14 - of the structures \\
\hline $1_{8}\left(\mathrm{DMSO}-\mathrm{D}_{2} \mathrm{O}\right)$ & 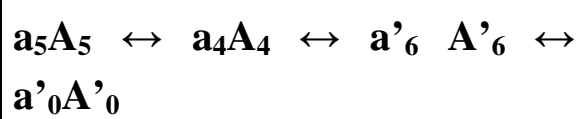 & $7.532-7.395$ & $\mathbf{a}_{1} \mathbf{A}_{1} \leftrightarrow \mathbf{a}^{\prime} \mathbf{A}^{\prime}{ }_{1} \leftrightarrow$ \\
\hline $1_{2}(\mathrm{DM}$ & $\begin{array}{l}\mathbf{a}_{7} \mathbf{A}^{\prime}{ }_{7} \leftrightarrow \mathbf{a}^{\prime} \mathbf{A}^{\prime}{ }_{1} \leftrightarrow \mathbf{a}^{\prime} \mathbf{A}^{\prime}{ }_{6} \leftrightarrow \\
\mathbf{a}^{\prime} \mathbf{A}^{\prime} \mathbf{A}_{0}\end{array}$ & $7.517-7.381$ & $\mathbf{a}_{2} \mathbf{A}_{2} \leftrightarrow \mathbf{a}_{3} \mathbf{A}^{\prime}{ }_{3} \leftrightarrow$ \\
\hline $1_{1}(\mathrm{DMSO})$ & $a^{\prime}{ }_{7}^{\prime}{ }^{\prime} \leftrightarrow a^{\prime}{ }_{4} A^{\prime}{ }_{4} \leftrightarrow a^{\prime}{ }_{0} A^{\prime}{ }_{0}$ & $7.503-7.336$ & $\mathbf{a}_{2} \mathbf{A}_{2} \leftrightarrow \mathbf{a}^{\prime}{ }_{4} \mathbf{A}^{\prime}{ }_{4} \leftrightarrow \mathbf{a A}$ \\
\hline $1_{3}(\mathrm{C}$ & $\mathbf{a}^{\prime}{ }_{4} \mathbf{A}^{\prime}{ }_{4} \leftrightarrow \mathbf{a}^{\prime}{ }_{2} \mathbf{A}^{\prime}{ }_{2} \leftrightarrow \mathbf{a}_{1} \mathbf{A}_{1}$ & $7.349-7.212$ & $\mathbf{a}_{3}{ }_{3} \mathbf{\prime}_{3} \leftrightarrow \mathbf{a}^{\prime} \mathbf{A}^{\prime}$ \\
\hline $1_{4}(\mathrm{C}$ & $a^{\prime} A^{\prime}{ }^{\prime}{ }_{4} \leftrightarrow a^{\prime}{ }_{1} A^{\prime}{ }_{1} \leftrightarrow a^{\prime} A^{\prime}{ }_{0}$ & $7.342-7.205$ & $a^{\prime}{ }_{4} A^{\prime}{ }_{4} \leftrightarrow a^{\prime} A^{\prime}$ \\
\hline $5\left(\mathrm{CDCl}_{3}\right)$ & $\begin{array}{l}\mathbf{a}_{7} \mathbf{A}^{\prime}{ }_{7} \leftrightarrow \mathbf{a}^{\prime} \mathbf{A}^{\prime}{ }_{4} \leftrightarrow \mathbf{a}_{{ }_{2}} \mathbf{A}^{\prime}{ }_{2} \leftrightarrow \\
\mathbf{a}^{\prime} \mathbf{A}_{1} \mathbf{A}_{1} \leftrightarrow \mathbf{a}^{\prime} \mathbf{A}^{\prime} \mathbf{A}_{5} \leftrightarrow \mathbf{a}^{\prime} \mathbf{A}^{\prime}{ }_{3}\end{array}$ & $7.397-7.143$ & 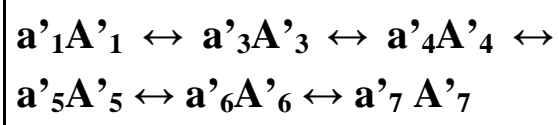 \\
\hline $4\left(\mathrm{CDCl}_{3}\right)$ & $a^{\prime} A^{\prime}{ }_{4} \leftrightarrow a^{\prime}{ }_{2} A^{\prime}{ }_{2} \leftrightarrow a^{\prime} A^{\prime}{ }^{\prime}{ }_{1}$ & $7.341-7.204$ & $\mathbf{a}^{\prime}{ }_{4} \mathbf{A}^{\prime}{ }_{4} \leftrightarrow \mathbf{a A}$ \\
\hline $1,2,6\left(\mathrm{CDCl}_{3}\right)$ & $\mathbf{a}_{2}{ }_{2} \mathbf{A}^{\prime}{ }_{2} \leftrightarrow \mathbf{a}_{5}{ }_{5} \mathbf{A}^{\prime}{ }_{5} \leftrightarrow \mathbf{a}_{0} \mathbf{A}^{\prime}{ }_{0}$ & $7.336-7.200$ & $\mathbf{a}^{\prime}{ }_{4} \mathbf{A}^{\prime}{ }_{4} \leftrightarrow \mathbf{a}^{\prime} \mathbf{A}^{\prime} \leftrightarrow \mathbf{a}^{\prime}{ }_{5} \mathbf{A}^{\prime}{ }_{5}$ \\
\hline $3\left(\mathrm{CDCl}_{3}\right)$ & $\mathbf{a}^{\prime} \mathbf{A}^{\prime}{ }_{1}{ }_{1} \leftrightarrow \mathbf{a}^{\prime}{ }_{5} \mathbf{A}^{\prime}{ }_{5}$ & $7.331-7.195$ & $\mathbf{a}_{5}{ } \mathbf{A}^{\prime}{ }_{5} \leftrightarrow \mathbf{a}^{\prime} \mathbf{A}^{\prime} \leftrightarrow \mathbf{a}^{\prime}{ }_{6} \mathbf{A}^{\prime}{ }_{6}$ \\
\hline
\end{tabular}

The H12 signals at 7.967 ppm - 7.869 ppm , $7.954 \mathrm{ppm}-7.859 \mathrm{ppm}$ and $7.935 \mathrm{ppm}-7.837 \mathrm{ppm}$ (spectra $1_{8} 1_{2} 1_{1}$, Tables 2, 5) indicate the $\mathbf{a}_{5} \mathbf{A}_{5} \leftrightarrow \mathbf{a}_{\mathbf{1}} \mathbf{A}_{\mathbf{1}} \leftrightarrow \mathbf{a}_{\mathbf{8}} \mathbf{A}_{\mathbf{8}} \leftrightarrow \mathbf{a} \mathbf{A}, \mathbf{a}_{\mathbf{8}} \mathbf{A}_{\mathbf{8}} \leftrightarrow \mathbf{a}_{7} \mathbf{A}^{\prime}{ }_{7}$ and $\mathbf{a}_{7}$ $\mathbf{A}^{\prime}{ }_{7} \leftrightarrow \mathbf{a}_{6} \mathbf{A}_{\mathbf{6}}$ resonance structures, respectively.

The H12 signals at $7.859 \mathrm{ppm}-7.688 \mathrm{ppm}$ and $7.854 \mathrm{ppm}-7.681 \mathrm{ppm}$ (spectra $1_{3} 1_{4}$, Tables 2, 5) support the resonance structures $\mathbf{a}_{7} \mathbf{A}^{\prime} \mathbf{7}_{\mathbf{7}} \leftrightarrow \mathbf{a}_{\mathbf{1}} \mathbf{A}_{\mathbf{1}} \leftrightarrow \mathbf{a} \mathbf{A}$ and $\mathbf{a}_{\mathbf{1}} \mathbf{A}_{\mathbf{1}} \leftrightarrow \mathbf{a}_{\mathbf{2}} \mathbf{A}^{\prime} \mathbf{2}_{\mathbf{2}} \leftrightarrow \mathbf{a}^{\prime} \mathbf{A}^{\prime}$, respectively. The $\mathrm{H} 12$ signals at $7.852 \mathrm{ppm}-7.683 \mathrm{ppm}$ and $7.852 \mathrm{ppm}-7.678 \mathrm{ppm}$ (spectra 4, 6, Tables 3, 5) confirm the resonance structures $\mathbf{a}_{\mathbf{2}} \mathbf{A}_{\mathbf{2}} \leftrightarrow \mathbf{a}_{\mathbf{2}} \mathbf{A}^{\mathbf{\prime}_{\mathbf{2}}} \leftrightarrow \mathbf{a} \mathbf{A}$ and $\mathbf{a}_{\mathbf{2}} \mathbf{A}_{\mathbf{2}} \leftrightarrow \mathbf{a}_{1} \mathbf{A}^{\prime}{ }_{1}$, respectively. The H12 signals at 7.847 ppm - 7.674 ppm and $7.838 \mathrm{ppm}-7.646 \mathrm{ppm}$ (spectra 1 - 3, 5, Tables 3, 5) confirm

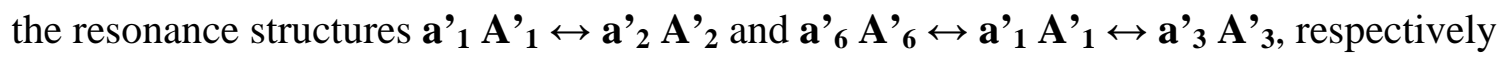

The calculated chemical shift of N10 at -86.0 ppm of 2a tautomer (Table 1) [10] point to an amine - type nitrogen atom. 
Table 7. The ${ }^{1} \mathrm{H}-\mathrm{NMR}$ chemical shifts $\delta$ [ppm] from TMS of 1.

\begin{tabular}{|c|c|c|}
\hline \multirow{2}{*}{$\begin{array}{l}\text { Spectrum No } \\
\text { (Solvent) }\end{array}$} & \multicolumn{2}{|r|}{ Pyridin - 2- yl } \\
\hline & H 11 & structures \\
\hline $1_{2}(\mathrm{DMSO})$ & $8.665-8.589$ & $\mathbf{a}_{4} \mathbf{A}_{4} \leftrightarrow \mathbf{a}^{\prime}{ }_{4} \mathbf{A}^{\prime}{ }_{4} \leftrightarrow \mathbf{a}^{\prime}{ }_{7} \mathbf{A}^{\prime}{ }_{7} \leftrightarrow \mathbf{a A}$ \\
\hline $1_{8}\left(\mathrm{DMSO}-\mathrm{D}_{2} \mathrm{O}\right)$ & $8.662-8.586$ & $\mathbf{a}_{4} \mathbf{A}_{4} \leftrightarrow \mathbf{a}_{6}{ }_{6} \mathbf{A}^{\prime}{ }_{6} \leftrightarrow \mathbf{a A}$ \\
\hline $1_{1}(\mathrm{DMSO})$ & $8.637-8.562$ & $\mathbf{a}_{3} \mathbf{A}_{3} \leftrightarrow \mathbf{a}_{5} \mathbf{A}_{5} \leftrightarrow \mathbf{a}^{\prime}{ }_{6} \mathbf{A}^{\prime}{ }_{6}$ \\
\hline $1_{3}\left(\mathrm{CDCl}_{3}\right)$ & $8.606-8.530$ & $\mathbf{a}_{7}{ }_{7} \mathbf{A}^{\prime}{ }_{7} \leftrightarrow \mathbf{a}^{\prime} \mathbf{A}^{\prime}{ }_{1} \leftrightarrow \mathbf{a}^{\prime}{ }_{8} \mathbf{A}^{\prime}{ }_{8} \leftrightarrow \mathbf{a} A$ \\
\hline $4\left(\mathrm{CDCl}_{3}\right)$ & $8.603-8.528$ & $\mathbf{a}^{\prime}{ }_{4} \mathbf{A}^{\prime}{ }_{4} \leftrightarrow \mathbf{a}_{5} \mathbf{A}^{\prime}{ }_{5} \leftrightarrow \mathbf{a A}$ \\
\hline $1_{4}\left(\mathrm{CDCl}_{3}\right)$ & $8.601-8.525$ & $\mathbf{a}_{6}{ }_{6} \mathbf{A}_{6}{ }_{\leftrightarrow} \leftrightarrow \mathbf{a}_{3} \mathbf{A}^{\prime}{ }_{3} \leftrightarrow \mathbf{a A}$ \\
\hline $3\left(\mathrm{CDCl}_{3}\right)$ & $8.598-8.537$ & $a^{\prime}{ }_{5} A^{\prime}{ }_{5} \leftrightarrow a_{8}{ }^{\prime} A^{\prime}{ }_{8} \leftrightarrow a A$ \\
\hline $6\left(\mathrm{CDCl}_{3}\right)$ & $8.598-8.523$ & $\mathbf{a}_{5}{ }_{5} \mathbf{\prime}_{5} \leftrightarrow \mathbf{a}^{\prime} \mathbf{A}^{\prime}$ \\
\hline $1\left(\mathrm{CDCl}_{3}\right)$ & $8.594-8.519$ & $a^{\prime}{ }_{5} A^{\prime}{ }_{5} \leftrightarrow a^{\prime}{ }_{3} A^{\prime}{ }_{3} \leftrightarrow a^{\prime}{ }_{0} A^{\prime}{ }_{0}$ \\
\hline $5\left(\mathrm{CDCl}_{3}\right)$ & $8.589-8.514$ & $\mathbf{a}_{3}{ }_{3} \mathbf{A}_{3} \leftrightarrow \mathbf{a}^{\prime} \mathbf{A}^{\prime}$ \\
\hline $2\left(\mathrm{CDCl}_{3}\right)$ & $8.580-8.537$ & $a^{\prime}{ }_{3} A^{\prime}{ }_{3} \leftrightarrow a^{\prime}{ }_{5} A^{\prime}{ }_{5} \leftrightarrow a^{\prime}{ }_{8} A^{\prime}{ }_{8} \leftrightarrow a A$ \\
\hline $5\left(\mathrm{CDCl}_{3}\right)$ & $8.387-8.345$ & $\mathbf{a}^{\prime}{ }_{1} \mathbf{A}^{\prime}{ }_{1} \leftrightarrow \mathbf{a}^{\prime}{ }_{2} \mathbf{A}^{\prime}{ }_{2} \leftrightarrow \mathbf{a}_{1} \mathbf{A}_{1}$ \\
\hline
\end{tabular}

The ${ }^{1} \mathrm{H}$ - data (100 MHz, $\left.500 \mathrm{MHz}\right),{ }^{13} \mathrm{C}$ - and ${ }^{15} \mathrm{~N}$-NMR spectra and the theoretical calculations of the studied system point to the transformation of pyridine - type nitrogen atom to pyrrole - type as well as to amine - type nitrogen of 1,3,4 - thiadiazole and pyridine rings, the structures a $\mathbf{A} \mathbf{A}(\mathbf{I}), \mathbf{a}_{0}$ $\mathbf{A}_{\mathbf{0}} \mathbf{A}(\mathbf{I})_{0}, \mathbf{a}^{\prime}{ }_{0} \mathbf{A}_{\mathbf{0}} \mathbf{A}(\mathbf{I})^{\prime}{ }_{0}$ and $\mathbf{a}$ ' A' A(I) ' (Figures 1-3).

The calculated chemical shift of NH group at 7.5 ppm of tautomer 2a (Table 1) [10] supports sp $^{2}$ hybridization of N6 as well as the absence of the charges over 1,3,4 thiadiazole ring. The calculated chemical shift of N6 at - 133.98 ppm of tautomer 2a (Table 1) [10] supports pyridine - type nitrogen atom.

In the ${ }^{1} \mathrm{H}-\mathrm{NMR}$ spectra of $\mathbf{1}(100 \mathrm{MHz})$ [7, 8] in the range of the chemical shifts of the proton of NH group from $8.637 \mathrm{ppm}$ to $7.233 \mathrm{ppm}$ the nitrogen atoms N3, N4, N10 appear as pyridine - type, pyrrole - type, amine - type nitrogen while N6 as pyridine - type A (Figures 5, 9 - 12). The absence of the charges over 1,3,4 - thiadiazole ring confirm the lack of the transition of electrons of $\mathrm{p}$ orbitals of 1S 2C 3N 4N 5C of the 1,3,4 - thiadiazole ring.

The changes concern either the phases of p orbitals of N3 N4 and N10 nitrogen atoms of 1,3,4 thiadiazole and pyridine rings, respectively see the structures $\mathbf{A}_{\mathbf{0 b}} \mathbf{A}_{\mathbf{0 d}}$ (Figure 11) or the spin states of electrons of $\mathrm{sp}^{2}$ orbitals, the structures $\mathbf{A}_{\mathbf{0}}, \mathbf{A}_{\mathbf{0}}$, (Figures 9,10 ) and support the transformation of pyridine - type nitrogen atom to pyrrole - type nitrogen atom in the second case. The simultaneous changes of the spin states of electrons of $\mathrm{sp}^{2}$ orbitals and of the phases of p orbitals of N3 N4 N10 nitrogen atoms of 1,3,4 - thiadiazole and pyridine rings as well as the absence of the reversed electron demand cause the transformation of pyridine nitrogen atom to amine nitrogen atom, the structures $\mathbf{A}_{\mathbf{0 c}} \mathbf{A}_{\mathbf{0 e}}$ (Figure 12). Consequently the structures $\mathbf{A}_{\mathbf{0}}, \mathbf{A}_{\mathbf{0 a}}$, (Figures 9, 10) show the pyrrole type nitrogen atoms N3 N4 N10 and the lack of the differences in the phases of p orbitals of 1S 2C 3N 4N 5C 6N 10N 11C - 15C. In the structures $\mathbf{A}_{\mathbf{0 b}} \mathbf{A}_{\mathbf{0 d}}$ (Figure 11) the nitrogen atoms N3 N4 N10 are the pyridine - type but the phases of p orbitals of N3 N10 and N4 N10 differ from p orbitals of 1S 2C 4N 5C 6N 11C - 15C and 1S 2C 3N 5C 6N 11C - 15C atoms, respectively. In the structures $\mathbf{A}^{\prime} \mathbf{A}_{\mathbf{a}}$ 
and $\mathbf{A}_{\mathbf{0}} \mathbf{A}_{\mathbf{0 a}}$ (Figures 13, 3) the nitrogen atom N10 is pyridine - type and p orbitals of N10 C11 C13 and N10 C11 C13 C14, respectively show no differences in their phases.

The ${ }^{1} \mathrm{H}-{ }^{1} \mathrm{H}$ long-range coupling constants in the $37.280 \mathrm{~Hz}-43.776 \mathrm{~Hz}$ range (spectra $1-6,6_{6}$ ) (Tables 8, 9) [8], support the coupling of the protons of the pyridyl and $-\mathrm{N}^{-} \mathrm{CH}_{2}-\mathrm{CH}=\mathrm{CH}_{2}$ groups via 2p orbitals of $\mathrm{C} 14 \mathrm{C} 7$ of the rigid structures $\mathbf{A}^{\prime} \mathbf{A}_{\mathbf{a}}$ and $\mathrm{sp}^{2}$ hybridization of the exocyclic nitrogen atom N6 (Figure 13). The signals at - 0.033 ppm - 5.787 ppm (Table 9, spectra 1, 3 - 5, 66 ) support the transformation of $\mathrm{sp}^{2} \Leftrightarrow \mathrm{sp}^{3}$.

The differences in the resonances of $\mathrm{NH}$ proton in the range from $8.637 \mathrm{ppm}$ to $7.233 \mathrm{ppm}$ are caused by the atomic charge over the pyridine ring. To assign the resonance structures of $\mathbf{1}$ in the range from $8.637 \mathrm{ppm}$ to $7.233 \mathrm{ppm}$ the chemical shifts of $\mathrm{NH}$ group, the ${ }^{15} \mathrm{~N}$-, ${ }^{13} \mathrm{C}$ - and ${ }^{1} \mathrm{H}$ - signals in ${ }^{15} \mathrm{~N}$-, ${ }^{13} \mathrm{C}$ - and ${ }^{1} \mathrm{H}-\mathrm{NMR}$ spectra $(100 \mathrm{MHz}, 500 \mathrm{MHz})$ of $\mathbf{1}$ as well as the ${ }^{1} \mathrm{H}-{ }^{1} \mathrm{H}$ coupling constants of the pyridyl substituent have been analyzed. The resonance structures of the pyridine ring are shown on Figure 8.

In the ${ }^{13} \mathrm{C}$-NMR spectrum of $\mathbf{1}$ the chemical shifts of C11 at $149.31 \mathrm{ppm}$ and C15 at $149.87 \mathrm{ppm}$

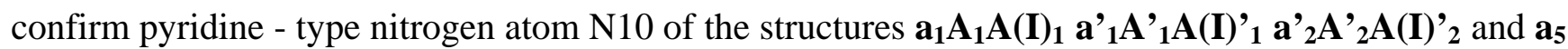
$\mathbf{A}_{5} \mathbf{A}(\mathbf{I})_{5}$, respectively. The chemical shift of $\mathrm{C} 12$ at $124.01 \mathrm{ppm}$ supports the pyridine - type nitrogen atom N10 of the structures $\mathbf{a}_{2} \mathbf{A}_{2} \mathbf{A}(\mathbf{I})_{2} \mathbf{a}_{3} \mathbf{A}^{\prime}{ }_{3} \mathbf{A}(\mathbf{I}){ }_{3} \mathbf{a}^{\prime}{ }_{5} \mathbf{A}{ }_{5}{ }_{5} \mathbf{A}(\mathbf{I}){ }^{5}$. The signal of C14 at $119.87 \mathrm{ppm}$ points to the structures $\mathbf{a}_{3} \mathbf{A}_{3} \mathbf{A}(\mathbf{I})_{3} \mathbf{a}_{4}{ }_{4} \mathbf{A}^{\prime}{ }_{4} \mathbf{A}(\mathbf{I}){ }^{{ }_{4}} \mathbf{a}_{5} \mathbf{A}_{5} \mathbf{A}(\mathbf{I})_{5}$. The signal of $\mathrm{C} 13$ at $136.77 \mathrm{ppm}$ confirms the structures $\mathbf{a}_{2} \mathbf{A}_{2} \mathbf{A}(\mathbf{I})_{2} \mathbf{a}_{3} \mathbf{A}^{\prime}{ }_{3} \mathbf{A}(\mathbf{I}){ }_{3} \mathbf{a}_{4} \mathbf{A}_{4} \mathbf{A}(\mathbf{I})_{4} \mathbf{a}_{5} \mathbf{A}^{\prime}{ }_{5} \mathbf{A}(\mathbf{I})^{\prime}{ }_{5}$.

Figure 9. The changes of the electron configuration of N4 N10 nitrogen atoms of allyl(5-pyridin-2- yl-[1,3,4] thiadiazol-2-yl)-amine, the structure $\mathbf{A}_{\mathbf{0}}$

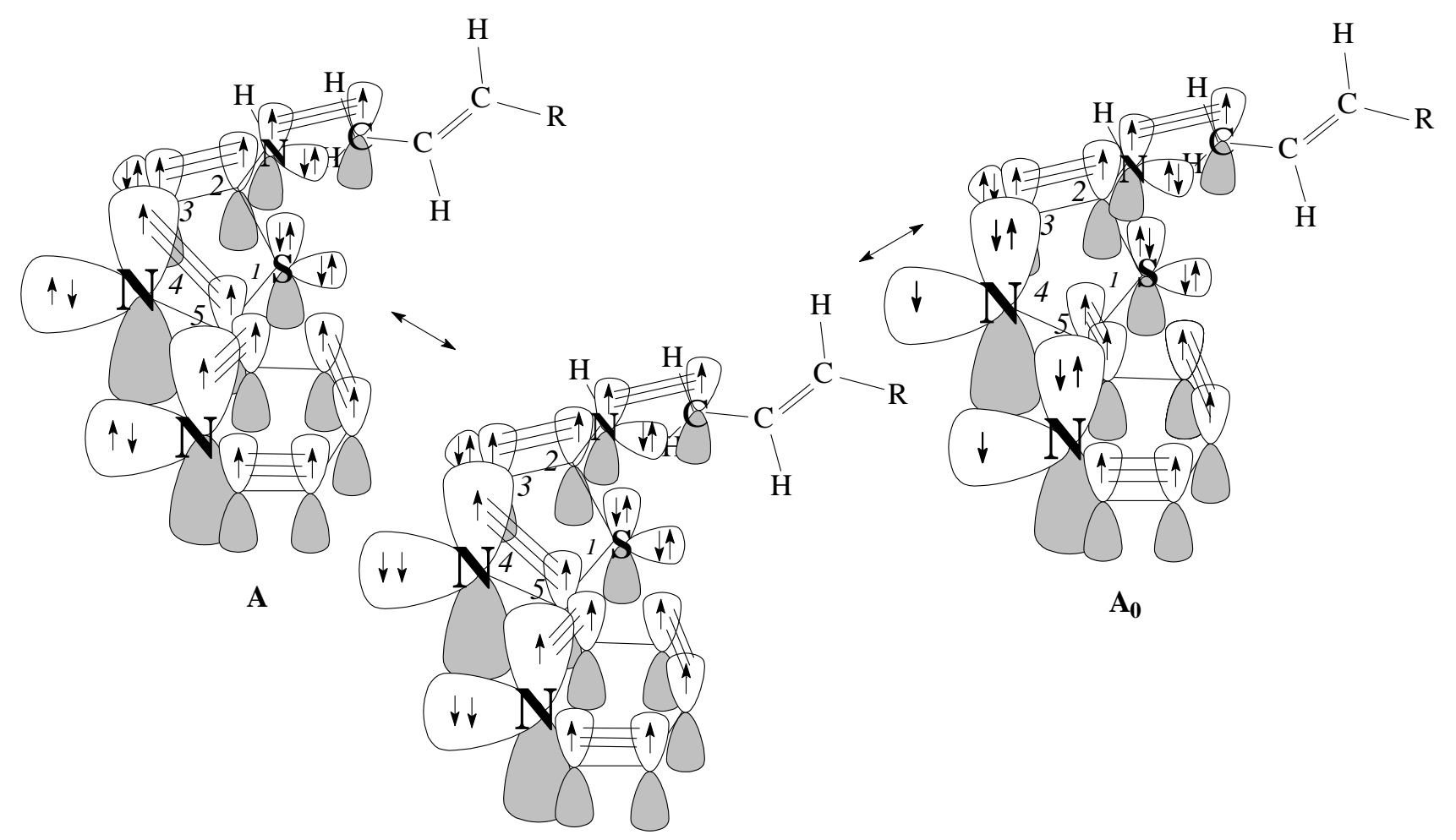


Figure 10. The changes of the electron configuration of N3 N10 nitrogen atoms of allyl- (5pyridin - 2 - yl - [1,3,4] thiadiazol-2-yl)-amine, the structure $\mathbf{A}_{\mathbf{0 a}}$

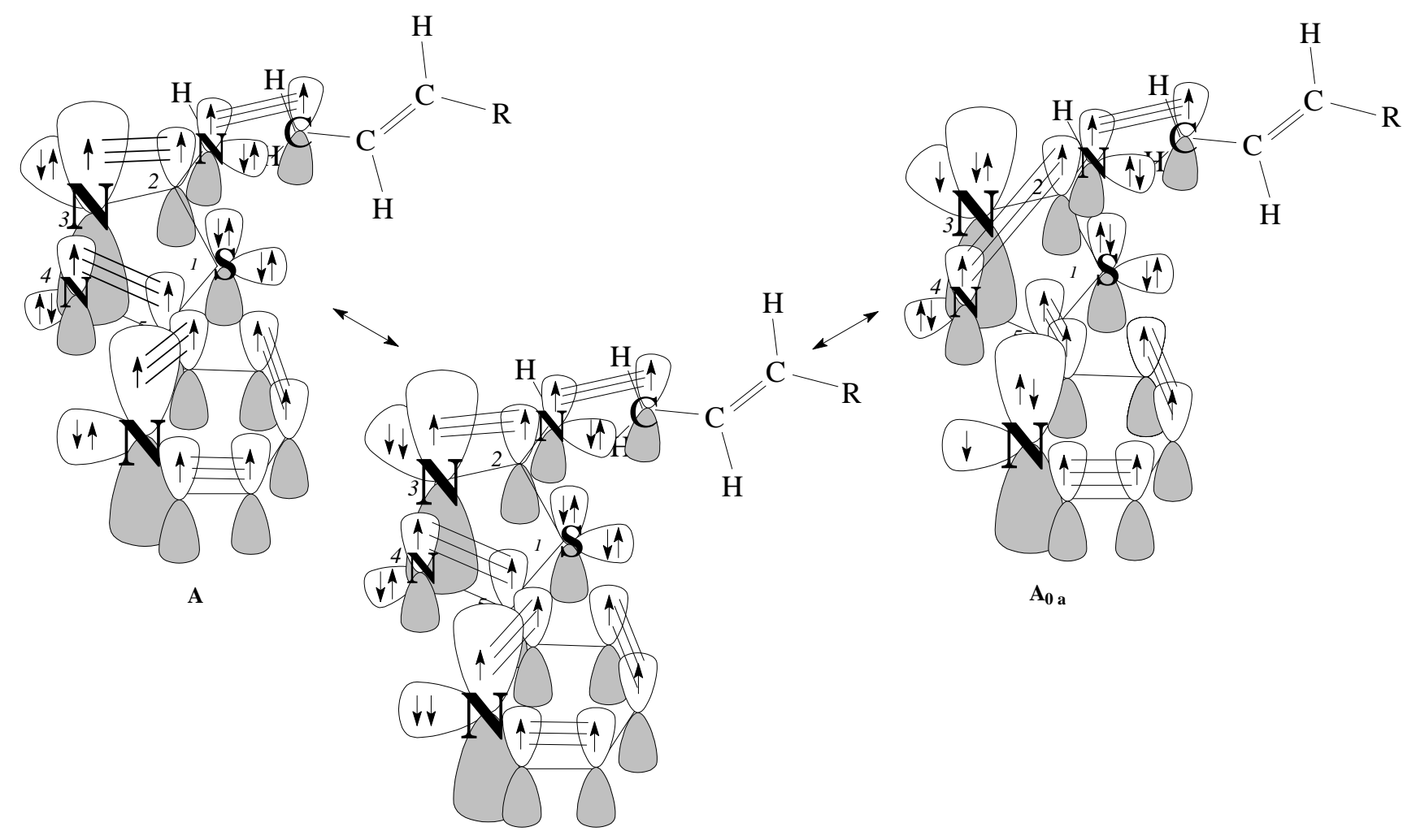

Figure 11. The changes of the electron configuration of N3 N10 and N4 N10 nitrogen atoms of allyl- (5-pyridin - 2 - yl - [1,3,4] thiadiazol - 2 - yl) - amine, the structures $\mathbf{A}_{\mathbf{0 b}} \mathbf{A}_{\mathbf{0 d}}$

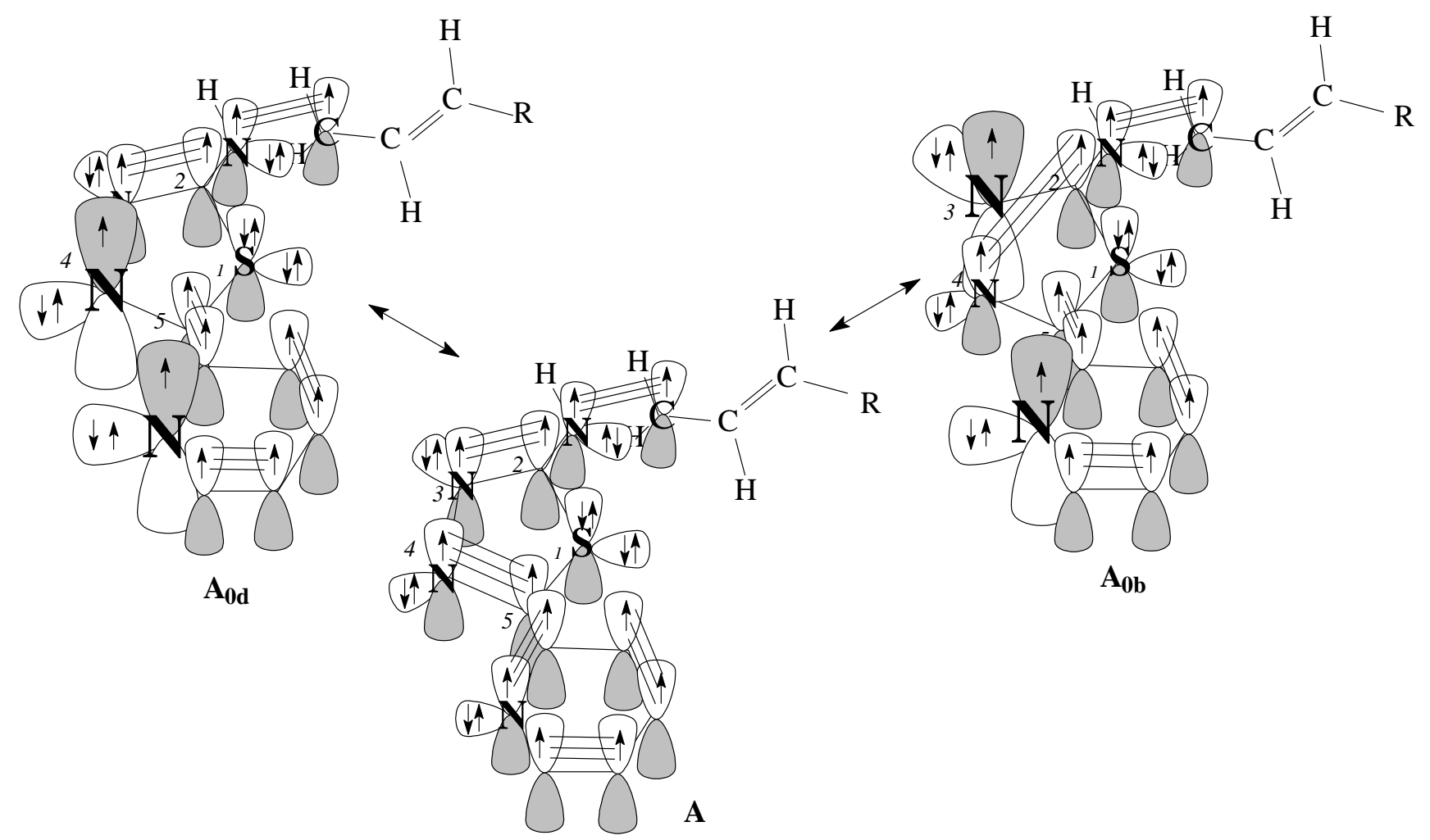


Figure 12. The changes of the electron configuration of N3 N10 and N4 N10 nitrogen atoms of allyl- (5-pyridin - 2 - yl - [1,3,4] thiadiazol-2-yl)-amine, the structures $\mathbf{A}_{\mathbf{0 c}} \mathbf{A}_{\mathbf{0 e}}$

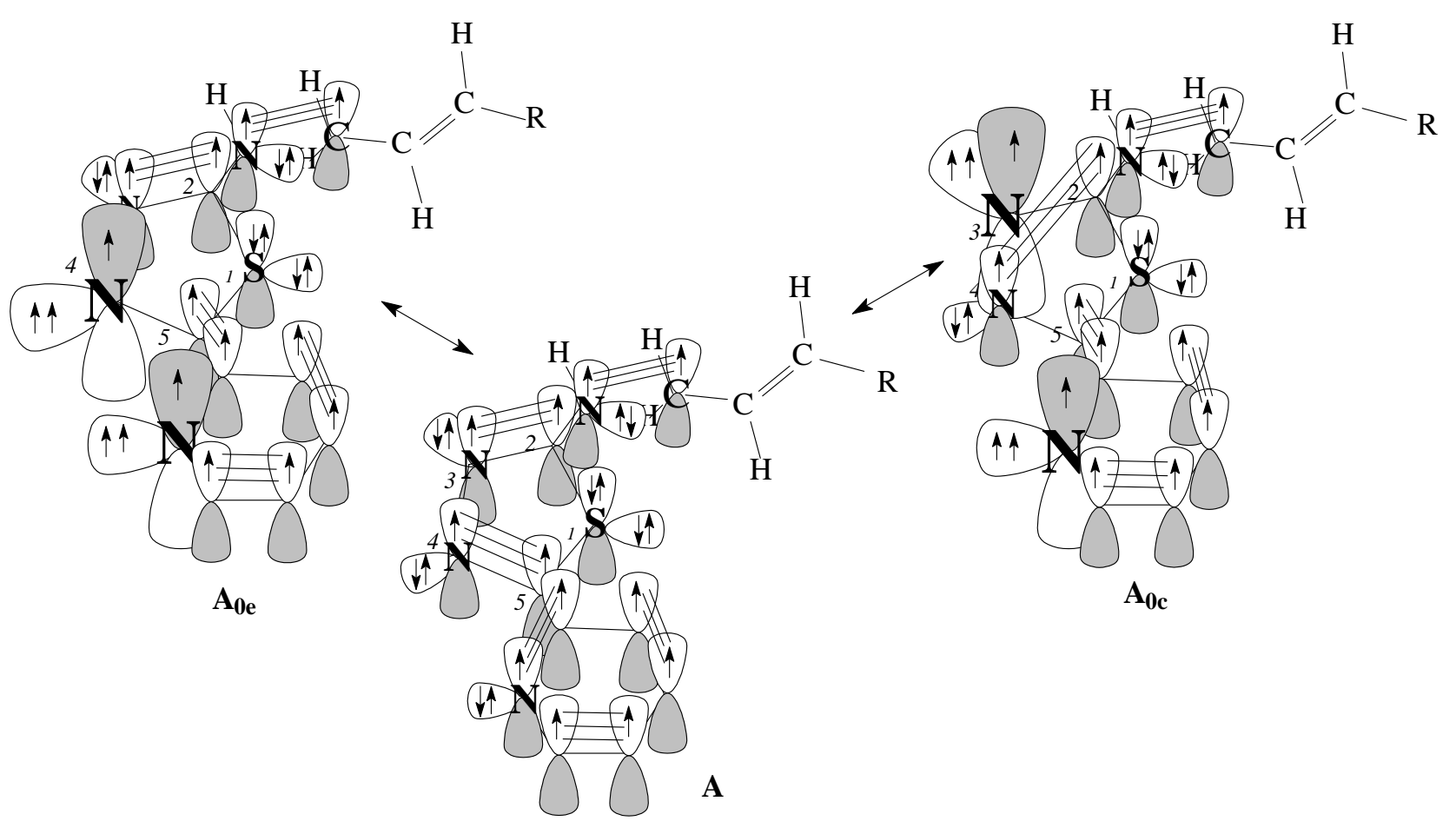

Figure 13. The resonance rigid structures $\mathbf{A}^{\prime}, \mathbf{A}_{\mathbf{a}}$ of allyl- (5-pyridin $-2-\mathrm{yl}-[1,3,4]$ thiadiazol-2-yl)-amine
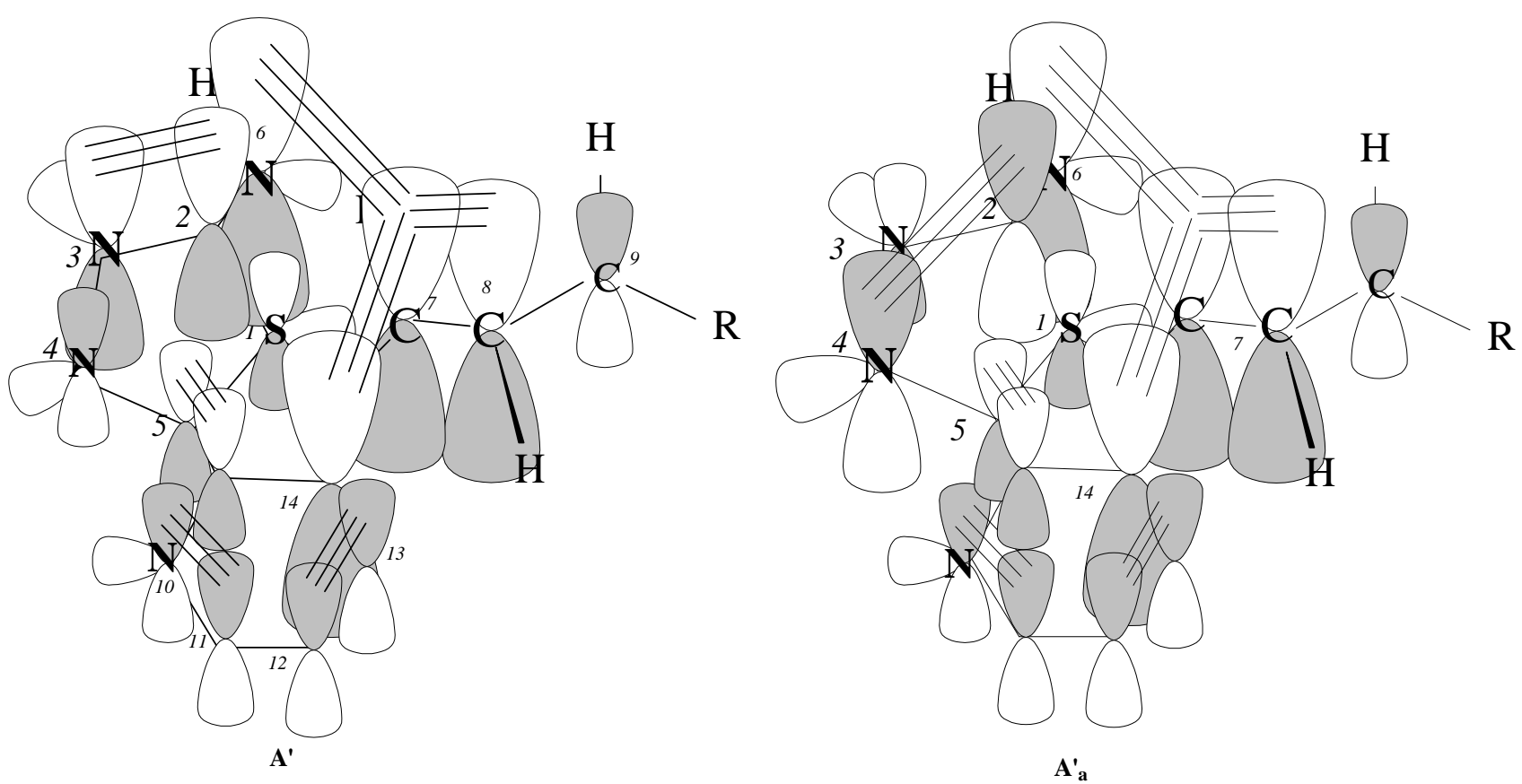

The ${ }^{1} \mathrm{H}-\mathrm{NMR}$ spectrum $1_{7}(500 \mathrm{MHz})$ shows the signal of $\mathrm{H} 14$ of the structures $\mathbf{a}^{\prime}{ }_{\mathbf{1}} \mathbf{A}^{{ }_{1}} \mathbf{A}(\mathbf{I}){ }_{1} \mathbf{a} \mathbf{a}_{\mathbf{5}}$

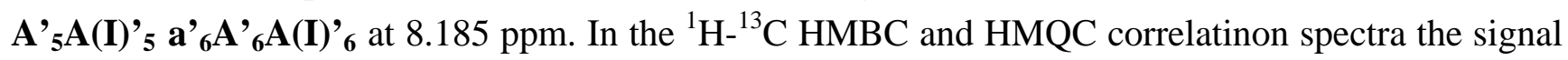
of $\mathrm{H} 14$ at 8.180 ppm exhibits a correlation to C14 at 119.7 ppm and C12 at 124.0 ppm, C15 at 149.7 ppm, C5 at $160.0 \mathrm{ppm}$, respectively and confirms $\mathbf{a}{ }_{5} \mathbf{A}_{5}{ }_{5} \mathbf{A}(\mathbf{I}){ }_{5} \mathbf{a}_{6} \mathbf{A}_{\mathbf{6}} \mathbf{A}{ }_{6} \mathbf{A}(\mathbf{I}){ }_{6}{ }_{6}$ structures. In the $2 \mathrm{D}{ }^{1} \mathrm{H}-$ ${ }^{13} \mathrm{C}$ HMQC spectra the cross - peak between $\mathrm{H} 11$ at 8.340 ppm and C14 at 119.9 ppm as well as the 
correlation signals of $\mathrm{H} 11$ at $8.360 \mathrm{ppm}$ to C14 at $119.9 \mathrm{ppm}, \mathrm{C} 15$ at 149,7 ppm support structures a'2 $\mathbf{A}^{\prime}{ }_{2} \mathbf{A}(\mathbf{I}){ }^{\prime}{ }_{2} \mathbf{a}_{\mathbf{1}} \mathbf{A}_{\mathbf{1}} \mathbf{A}(\mathbf{I})_{\mathbf{1}}$. The chemical shift of N10 in ${ }^{15} \mathrm{~N}$-NMR spectrum of $\mathbf{1}$ at -74.78 ppm supports

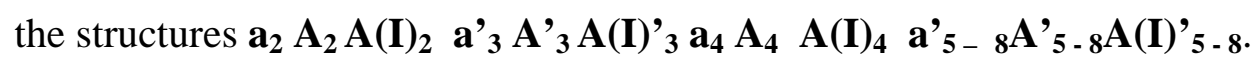

The ${ }^{1} \mathrm{H}-{ }^{1} \mathrm{H}$ coupling constants $\mathrm{J}\left(\mathrm{H}_{14} \mathrm{H}_{13}\right) 8.0 \mathrm{~Hz} \mathrm{~J}\left(\mathrm{H}_{13} \mathrm{H}_{14}\right) 8.0 \mathrm{~Hz} \mathrm{~J}\left(\mathrm{H}_{12} \mathrm{H}_{13}\right)$ 8.0 Hz of 1a tautomer confirm the positive charge at $\mathrm{C} 13$ atom of the structures $\mathbf{a}_{3} \mathbf{A}_{3} \mathbf{A}(\mathbf{I})_{3} \quad \mathbf{a}^{{ }^{\prime}}{ }_{4} \mathbf{A}^{\prime}{ }_{4} \mathbf{A}(\mathbf{I}){ }^{\prime}{ }_{4}$ while the coupling constants $\mathrm{J}\left(\mathrm{H}_{12} \mathrm{H}_{13}\right) 5.8 \mathrm{~Hz} \mathrm{~J}\left(\mathrm{H}_{11} \mathrm{H}_{12}\right) 5.6 \mathrm{~Hz} \mathrm{~J}\left(\mathrm{H}_{13} \mathrm{H}_{11}\right) 1.6 \mathrm{~Hz}$ indicate the positive charge at $\mathrm{C} 15$ and the negative one at $\mathrm{N} 10$ atoms of pyridine substituent of the structures $\mathbf{a}_{4} \mathbf{A}_{\mathbf{4}} \mathbf{A}(\mathbf{I})_{\mathbf{4}} \mathbf{a}^{\mathbf{}_{7}} \mathbf{A}(\mathbf{I})^{\mathbf{y}_{7}}$.

In the ${ }^{1} \mathrm{H}$-NMR $(100 \mathrm{MHz})$ spectra $1_{1}-4,1-6$, the $\mathrm{NH}$ group signals in the $8.637 \mathrm{ppm}$ to 8.514 ppm and $8.387 \mathrm{ppm}-8.138 \mathrm{ppm}$ range confirm the resonance structures $\mathbf{1 A}, \mathbf{1} \mathbf{A}^{\prime}, \mathbf{1} \mathbf{A}_{1}, \mathbf{1} \mathbf{A}_{\mathbf{2}}$ and $\mathbf{1} \mathbf{A}_{3}$, $\mathbf{1 A}_{\mathbf{4}}, \mathbf{1} \mathbf{A}_{\mathbf{5}}$ respectively (Table 10). At $8.077 \mathrm{ppm}-7.646 \mathrm{ppm}$ and at $7.397 \mathrm{ppm}-7.143 \mathrm{ppm} \mathbf{1 \mathbf { A } ^ { \prime }}{ }_{\mathbf{1}}$,

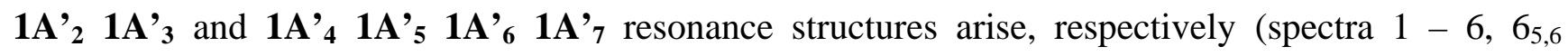
$100 \mathrm{MHz}, \mathrm{CDCl}_{3}$, Table 11). The signals at $8.594 \mathrm{ppm} J\left(\mathrm{H}_{11} \mathrm{H}_{9 \mathrm{~B}}\right) 42.432 \mathrm{~Hz}, 8.584 \mathrm{ppm} J\left(\mathrm{H}_{11} \mathrm{H}_{9 \mathrm{~A}}\right)$ $38.400 \mathrm{~Hz}, 8.528 \mathrm{ppm} J\left(\mathrm{H}_{11} \mathrm{H}_{9 \mathrm{~A}}\right) 37.280 \mathrm{~Hz}$ and $7.998 \mathrm{ppm} J\left(\mathrm{H}_{13} \mathrm{H}_{9 \mathrm{~A}}\right)$ 40.064 Hz (spectra 4, 5 Table 8) point to the transition of $\mathbf{A}^{\mathbf{\prime}} \Leftrightarrow \mathbf{A}$ and $\mathbf{A}^{\mathbf{\prime}}{ }_{\mathbf{1}} \Leftrightarrow \mathbf{A}_{\mathbf{1}}$ tautomers as well as to the rapid exchange at the $\mathrm{NH}$ group hydrogen of structure $\mathbf{A}$.

Table 8. The ${ }^{1} \mathrm{H}-\mathrm{NMR}$ chemical shifts $\delta[\mathrm{ppm}]$ from TMS and the ${ }^{1} \mathrm{H}-{ }^{1} \mathrm{H}$ long - range coupling constants [Hz] of $\mathbf{1}$

\begin{tabular}{|c|c|c|c|}
\hline $\begin{array}{c}\text { Spectrum } \\
\text { No }\left(\mathrm{CDCl}_{3}\right)\end{array}$ & $\delta(\mathrm{ppm})$ & $\boldsymbol{J}$ & NH \\
\hline 4 & 8.528 & $J\left(\mathrm{H}_{11} \mathrm{H}_{9 \mathrm{~A}}\right) 37.280$ & \\
\hline 6 & 8.598 & $J\left(\mathrm{H}_{11} \mathrm{H}_{9 \mathrm{~A}}\right) 38.144$ & $0.1 \mathrm{H}$ \\
\hline 1 & 7.754 & $J\left(\mathrm{H}_{12} \mathrm{H}_{9 \mathrm{~A}}\right) 38.336$ & $0.43 \mathrm{H}$ \\
\hline 4 & 8.584 & $J\left(\mathrm{H}_{11} \mathrm{H}_{9 \mathrm{~A}}\right) 38.400$ & \\
\hline 6 & 7.852 & $J\left(\mathrm{H}_{12} \mathrm{H}_{9 \mathrm{~A}}\right) 38.912$ & $0.14 \mathrm{H}$ \\
\hline 3 & 6.012 & $J\left(\mathrm{H}_{8} \mathrm{H}_{13}\right) \quad 40.832$ & $0.019 \mathrm{H}$ \\
\hline 3 & 5.895 & $J\left(\mathrm{H}_{8} \mathrm{H}_{13}\right) \quad 42.368$ & \\
\hline 3 & 5.886 & $J\left(\mathrm{H}_{8} \mathrm{H}_{14}\right) \quad 39.168$ & \\
\hline 5 & 7.974 & $J\left(\mathrm{H}_{13} \mathrm{H}_{9 \mathrm{~A}}\right) 39.296$ & $0.756 \mathrm{H}$ \\
\hline 4 & 7.331 & $J\left(\mathrm{H}_{14} \mathrm{H}_{9 \mathrm{~A}}\right) 39.392$ & $0.46 \mathrm{H}$ \\
\hline 4 & 7.341 & $J\left(\mathrm{H}_{14} \mathrm{H}_{9 \mathrm{~A}}\right) 40.640$ & \\
\hline 2 & 6.008 & $J\left(\mathrm{H}_{8} \mathrm{H}_{12}\right) \quad 39.872$ & $0.071 \mathrm{H}$ \\
\hline 2 & 5.890 & $J\left(\mathrm{H}_{8} \mathrm{H}_{13}\right) \quad 41.728$ & \\
\hline 6 & 5.839 & $J\left(\mathrm{H}_{8} \mathrm{H}_{12}\right) 39.936$ & $0.03 \mathrm{H}$ \\
\hline 5 & 7.998 & $J\left(\mathrm{H}_{13} \mathrm{H}_{9 \mathrm{~A}}\right) 40.064$ & \\
\hline 1 & 8.152 & $J\left(\mathrm{H}_{13} \mathrm{H}_{9 \mathrm{~A}}\right) 40.672$ & $0.38 \mathrm{H}$ \\
\hline 5 & 7.819 & $J\left(\mathrm{H}_{12} \mathrm{H}_{9 \mathrm{~A}}\right) 40.832$ & $1.356 \mathrm{H}$ \\
\hline 1 & 8.223 & $J\left(\mathrm{H}_{13} \mathrm{H}_{9 \mathrm{~A}}\right) 41.760$ & $0.38 \mathrm{H}$ \\
\hline 6 & 7.697 & $J\left(\mathrm{H}_{12} \mathrm{H}_{9 \mathrm{~A}}\right) 41.984$ & $0.14 \mathrm{H}$ \\
\hline 6 & 8.218 & $J\left(\mathrm{H}_{13} \mathrm{H}_{9 \mathrm{~B}}\right) 42.240$ & $0.172 \mathrm{H}$ \\
\hline 4 & 8.594 & $J\left(\mathrm{H}_{11} \mathrm{H}_{9 \mathrm{~B}}\right) 42.432$ & \\
\hline 5 & 8.223 & $J\left(\mathrm{H}_{13} \mathrm{H}_{9 \mathrm{~B}}\right) 43.776$ & $0.633 \mathrm{H}$ \\
\hline
\end{tabular}


In the ${ }^{1} \mathrm{H}-\mathrm{NMR}$ spectra $1_{1} 1_{2}$ (100MHz, DMSO) [8] the signals at $8.270 \mathrm{ppm}$ (1.08 $\mathrm{H}$, broadened triplet) and at $8.310 \mathrm{ppm}(1.05 \mathrm{H}$, degenerated broadened triplet) (Table 10) correspond to the $\mathrm{NH}$ group proton of tautomers $\mathbf{1 A}_{\mathbf{5}}$ and $\mathbf{1} \mathbf{A}_{\mathbf{4}}$, respectively. The broaded triplets suggest that these protons take part in the intermolecular hydrogen bonds. The broaded triplet in the ${ }^{1} \mathrm{H}-\mathrm{NMR}$ spectrum $1_{1}$ indicates the slow exchange of the proton of $\mathrm{NH}$ group, due to this fact, the coupling of the protons $\mathrm{H} 6$ H7 may be observed and support 1A tautomer. These signals are the averaged ones in consequence of the rapid transitions of hydrogen atom between the exocyclic nitrogen atom N6 and N3 N4 ones of 1,3,4-thiadiazole ring, then degenerated broaded triplet at $8.310 \mathrm{ppm}$ in the ${ }^{1} \mathrm{H}$-NMR spectrum $1_{2}$ point to the $\mathbf{1 A}_{\mathbf{4}} \mathbf{1 B}_{\mathbf{4}} \mathbf{1 C}_{\mathbf{4}}$ tautomers.. They disappear in $\mathrm{D}_{2} \mathrm{O}$ (spectrum $1_{8}$ ) .

Table 9. The ${ }^{1} \mathrm{H}-\mathrm{NMR}$ chemical shifts $\delta$ [ppm] from TMS and the ${ }^{1} \mathrm{H}-{ }^{1} \mathrm{H}$ long range coupling constants [Hz] of $\mathbf{1}$.

\begin{tabular}{|c|c|c|c|}
\hline $\begin{array}{c}\text { Spectrum No. } \\
\left(\mathbf{C D C l}_{\mathbf{3}}\right)\end{array}$ & $\boldsymbol{\delta}$ & $\boldsymbol{J}$ & $\mathbf{N H}$ \\
\hline 1 & 3.999 & $J\left(\mathrm{H}_{7 \mathrm{D}} \mathrm{H}_{11}\right) 37.696$ & $0.822 \mathrm{H}$ \\
3 & -0.033 & $J\left(\mathrm{H}_{6} \mathrm{H}_{11}\right) 38.272$ & $0.099 \mathrm{H}$ \\
3 & 5.477 & $J\left(\mathrm{H}_{9 \mathrm{~A}} \mathrm{H}_{13}\right) 40.192$ & $0.26 \mathrm{H}$ \\
3 & 5.787 & $J\left(\mathrm{H}_{8} \mathrm{H}_{14}\right) 43.136$ & \\
4 & 5.214 & $J\left(\mathrm{H}_{9 \mathrm{~B}} \mathrm{H}_{14}\right) 43.712$ & $0.24 \mathrm{H}$ \\
4 & 5.280 & $J\left(\mathrm{H}_{9 \mathrm{~A}} \mathrm{H}_{12}\right) 40.224$ & \\
5 & 5.266 & $J\left(\mathrm{H}_{9 \mathrm{~A}} \mathrm{H}_{12}\right) 40.960$ & $0.9 \mathrm{H}$ \\
5 & 5.449 & $J\left(\mathrm{H}_{9 \mathrm{~A}} \mathrm{H}_{13}\right) 39.680$ & \\
6 & 3.999 & $J\left(\mathrm{H}_{6} \mathrm{H}_{12}\right) 40.960$ & $0.183 \mathrm{H}$ \\
6 & 4.018 & $J\left(\mathrm{H}_{6} \mathrm{H}_{11}\right) 38.656$ & $0.19 \mathrm{H}$ \\
\hline
\end{tabular}

In ${ }^{1} \mathrm{H}-\mathrm{NMR}$ spectrum $5\left(100 \mathrm{MHz}, \mathrm{CDCl}_{3}\right)$ in the range from $7.397 \mathrm{ppm}$ to $7.143 \mathrm{ppm}$ a signal with an intensity of $7.5 \mathrm{H}$ is seen (Figure 14, Tables 3, 11).

Two of them correspond to the proton of the pyridine ring of the resonance structures $\mathbf{a}^{\prime} \mathbf{A}^{\prime} \mathbf{1}_{\mathbf{1}} \leftrightarrow \mathbf{a}^{\prime}{ }_{3}$

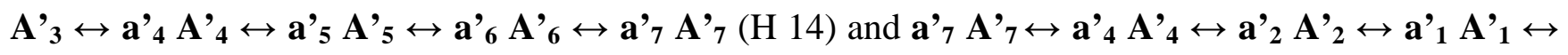
$\mathbf{a}_{5} \mathbf{A}_{5} \leftrightarrow \mathbf{a}_{3} \mathbf{A}_{3}{ }_{3}(\mathrm{H} \mathrm{12})$, (Tables 3, 6). The signal with the intensity of $5.5 \mathrm{H}$ correspond to the proton

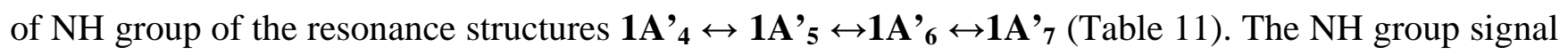
in the range from $7.397 \mathrm{ppm}$ to $7.265 \mathrm{ppm}$ with the intensity of $4.4 \mathrm{H}$ points to the transformation process of $\mathbf{1} \mathbf{A}^{\prime}{ }_{\mathbf{5}} \Rightarrow \mathbf{1 B}_{\mathbf{3}} \Rightarrow \mathbf{1 \mathbf { C } ^ { \prime }}{ }_{\mathbf{4}}$ tautomers and supports pyridine - type nitrogen atoms N10, N4, N6 (Figures 1, 2, 8). The signals at $7.331 \mathrm{ppm} \mathrm{J}\left(\mathrm{H}_{14} \mathrm{H}_{9 \mathrm{~A}}\right) 39.392 \mathrm{~Hz}$ and $7.341 \mathrm{~J}\left(\mathrm{H}_{14} \mathrm{H}_{9 \mathrm{~A}}\right) 40.640 \mathrm{~Hz}(0.46 \mathrm{H}$, Table 8. Spectrum 4) support the $\mathbf{1 A}^{\prime}{ }_{4} \mathbf{1 B}^{\prime}{ }_{4} \mathbf{1 C}{ }^{\prime}{ }_{4}$ tautomers. 
Figure 14. The ${ }^{1} \mathrm{H}-\mathrm{NMR}$ NH group signal at $7.397 \mathrm{ppm}-7.143 \mathrm{ppm}$ (spectrum 5)

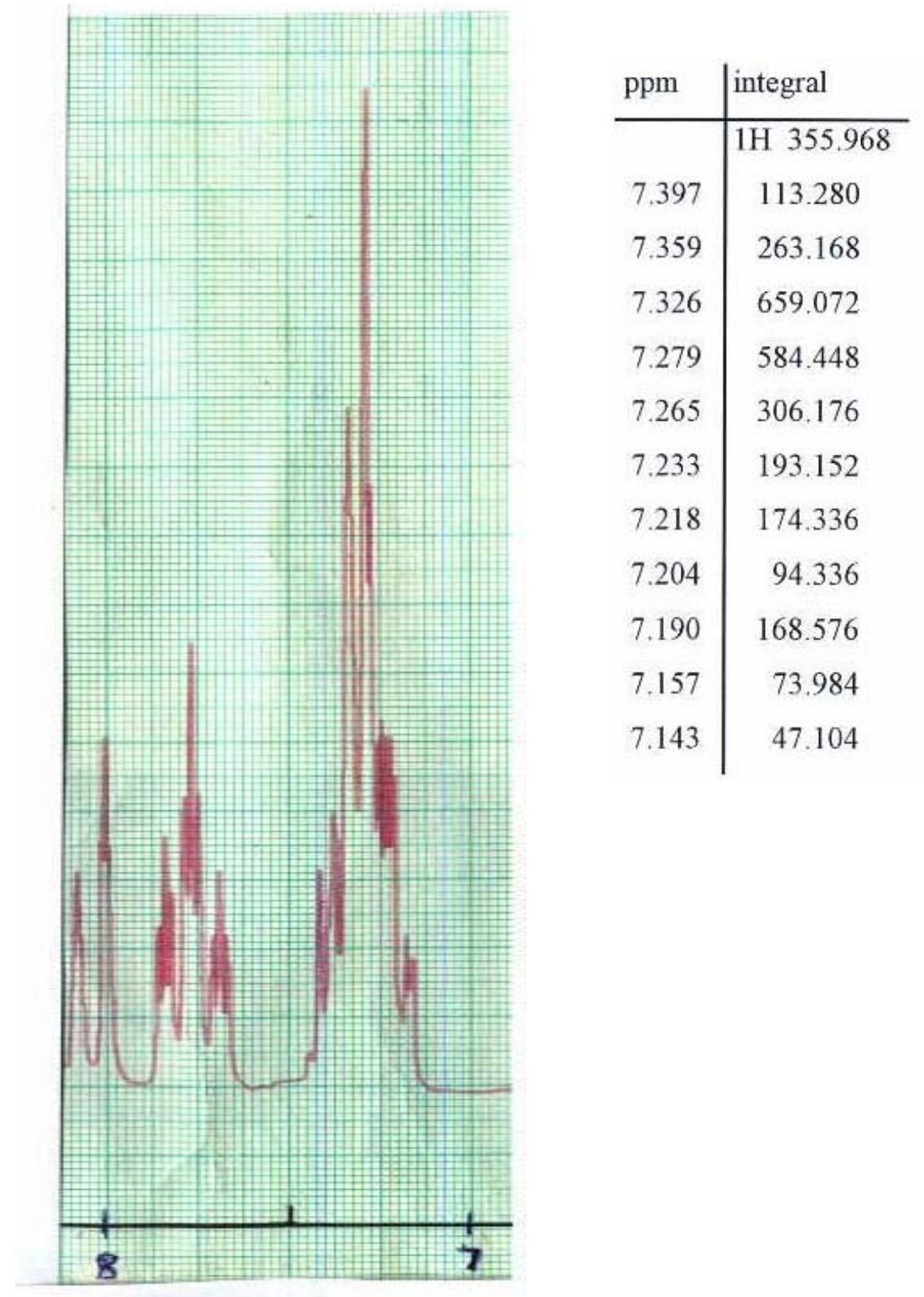

In ${ }^{1} \mathrm{H}-\mathrm{NMR}$ spectrum $6_{5}\left(100 \mathrm{MHz}, \mathrm{CDCl}_{3}\right)$ the signals at $7.317 \mathrm{ppm}(0.74 \mathrm{H})$ and $7.256 \mathrm{ppm}$ $(0.34 \mathrm{H})$ (Table 11$)$ correspond to the proton of $\mathbf{N H}$ group of $\mathbf{1} \mathbf{A}_{\mathbf{5}}$ and $\mathbf{1} \mathbf{A}_{\mathbf{6}} \mathbf{6}_{\text {tautomers. A signal at }}$ $7.233 \mathrm{ppm}(2 \mathrm{H})$ (the spectra $6_{5}$ and $6_{6} 100 \mathrm{MHz}, \mathrm{CDCl}_{3}$, Figure 15, Table 11) confirms the transformation process of $\mathbf{1 A}^{\mathbf{x}_{7}} \Rightarrow \mathbf{1 \mathbf { B } ^ { \prime }}{ }_{7}, \mathbf{1 A}^{\mathbf{x}_{7}} \Rightarrow \mathbf{1 \mathbf { C } ^ { \prime }}{ }_{7}$ tautomers and the amine - type nitrogen atoms N4, N3 of the 1,3,4-thiadiazole ring. 
Figure 15. The ${ }^{1} \mathrm{H}-\mathrm{NMR}$ NH group signal at $7.233 \mathrm{ppm}$ (spectra $66_{5}, 6_{6}$ )

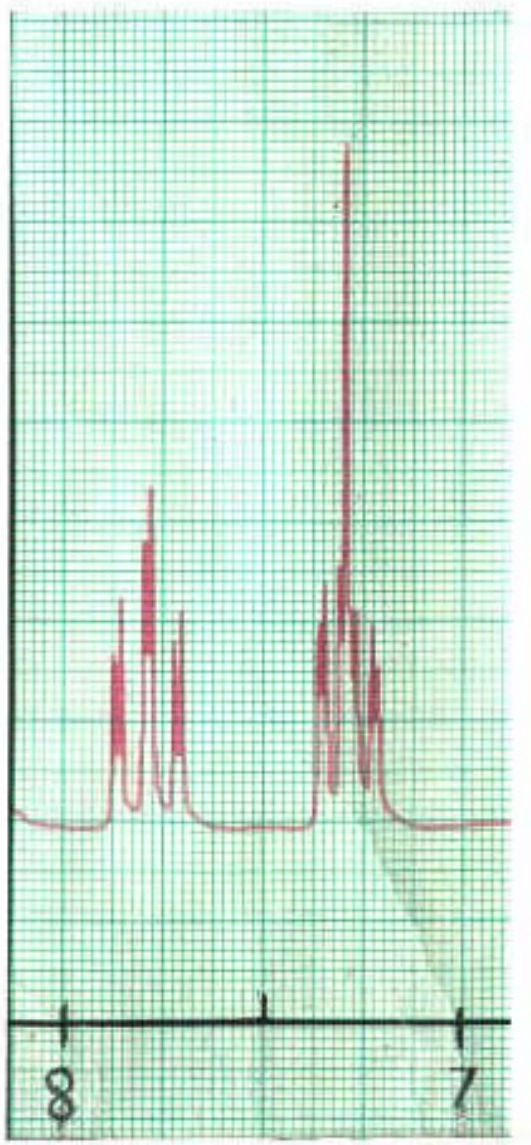

\begin{tabular}{c|c|c}
$\begin{array}{l}\text { Spectrum } \\
\text { No }\end{array}$ & ppm & integral \\
\hline $6_{5}$ & 7.233 & 1316.352 \\
$6_{6}$ & 7.233 & 801.792
\end{tabular}

Table 10. The ${ }^{1} \mathrm{H}-\mathrm{NMR}$ chemical shifts $\delta$ [ppm] from TMS of the NH group of tautomer $1 \mathrm{~A}$

\begin{tabular}{|c|c|c|}
\hline Spectrum No (Solvent) & NH & Structure \\
\hline $1_{1}(\mathrm{DMSO})$ & $8.637-8.562(0.08 \mathrm{H})$ & $\mathbf{1 A ~ 1 A} \mathbf{A}^{\prime}$ \\
$1_{3}\left(\mathrm{CDCl}_{3}\right)$ & $8.606-8.530(0.2 \mathrm{H})$ & $\mathbf{1 A}_{\mathbf{1}} \mathbf{1 A}_{\mathbf{2}}$ \\
$1_{4}\left(\mathrm{CDCl}_{3}\right)$ & $8.601-8.525(0.05 \mathrm{H})$ & \\
$3\left(\mathrm{CDCl}_{3}\right)$ & $8.598-8.537(0.23 \mathrm{H})$ & \\
$6\left(\mathrm{CDCl}_{3}\right)$ & $8.598-8.523(0.1 \mathrm{H})$ & \\
$1\left(\mathrm{CDCl}_{3}\right)$ & $8.594-8.519(0.38 \mathrm{H})$ & \\
$5\left(\mathrm{CDCl}_{3}\right)$ & $8.589-8.514(0.637 \mathrm{H})$ & \\
$2\left(\mathrm{CDCl}_{3}\right)$ & $8.580-8.537(0.08 \mathrm{H})$ & \\
$5\left(\mathrm{CDCl}_{3}\right)$ & $8.387-8.345(0.705 \mathrm{H})$ & $\mathbf{1 A}_{3}$ \\
$1_{2}(\mathrm{DMSO})$ & $8.310(1.05 \mathrm{H})$ & $\mathbf{1 A}_{\mathbf{4}}$ \\
$1_{1}(\mathrm{DMSO})$ & $8.270(1.08 \mathrm{H})$ & $\mathbf{1 A}_{\mathbf{5}}$ \\
$1\left(\mathrm{CDCl}_{3}\right)$ & $8.232-8.143(0.38 \mathrm{H})$ & \\
$2\left(\mathrm{CDCl}_{3}\right)$ & $8.237-8.148(0.1 \mathrm{H})$ & \\
$3\left(\mathrm{CDCl}_{3}\right)$ & $8.237-8.148(0.18 \mathrm{H})$ & \\
$4\left(\mathrm{CDCl}_{3}\right)$ & $8.242-8.152(0.07 \mathrm{H})$ & \\
$5\left(\mathrm{CDCl}_{3}\right)$ & $8.223-8.143(0.633 \mathrm{H})$ & \\
$6\left(\mathrm{CDCl}_{3}\right)$ & $8.228-8.138(0.172 \mathrm{H})$ & \\
$1_{7}\left(\mathrm{CDCl}_{3}\right)$ & $8.20-8.16(0.009 \mathrm{H})$ & \\
\hline
\end{tabular}


Table 11. The ${ }^{1} \mathrm{H}-\mathrm{NMR}$ chemical shifts $\delta$ [ppm] from TMS of the NH group of tautomer 1A' (in $\mathrm{CDCl}_{3}$ )

\begin{tabular}{|c|c|c|}
\hline Spectrum No & NH & Structure \\
\hline 5 & $8.077-7.974(0.756 \mathrm{H})$, & $1 \mathbf{A}^{\prime}{ }_{1}$ \\
\hline 4 & $7.852-7.683(0.13 \mathrm{H})$ & $1 \mathbf{A}^{\prime}{ }_{2}$ \\
\hline 6 & $7.852-7.678(0.14 \mathrm{H})$ & $1 \mathbf{A}^{\prime}{ }_{3}$ \\
\hline 1 & $7.847-7.674(0.43 \mathrm{H})$ & \\
\hline 2 & $7.847-7.674(0.18 \mathrm{H})$ & \\
\hline 3 & $7.847-7.674(0.25 \mathrm{H})$ & \\
\hline 5 & $7.838-7.646(1.356 \mathrm{H})$ & \\
\hline $1_{7}$ & $7.78-7.73(0.505 \mathrm{H})$ & \\
\hline 5 & $7.397-7,143(5.5 \mathrm{H})$ & $1 \mathbf{A}^{\prime}{ }_{4}$ \\
\hline 4 & $7.341-7.204(0.46 \mathrm{H})$ & $\mathbf{1 A}^{\mathbf{\prime}_{5}}$ \\
\hline 1 & $7.336-7.200(0.9 \mathrm{H})$ & $\mathbf{1 A}^{\mathbf{\prime}_{6}}$ \\
\hline 2 & $7.336-7.200(0.43 \mathrm{H})$ & $\mathbf{1 A}^{\prime}{ }_{7}$ \\
\hline 6 & $7.336-7.200(0.522 \mathrm{H})$ & \\
\hline 3 & $7.331-7.195(0.41 \mathrm{H})$ & \\
\hline $6_{5}$ & $7.317(0.74 \mathrm{H})$ & $\mathbf{1 \mathbf { A } ^ { \prime } { } _ { 5 }}$ \\
\hline $1_{7}$ & $7.29-7.25(0.3148 \mathrm{H})$ & $\mathbf{1 A}^{\mathbf{\prime}_{6}}$ \\
\hline $6_{5}$ & $7.256(0.34 \mathrm{H})$ & $\mathbf{1 A}^{\prime}{ }_{6}$ \\
\hline $6_{5}, 6_{6}$ & $7.233(2 \mathrm{H})$ & $\mathbf{1 A}^{\prime}{ }_{7}$ \\
\hline
\end{tabular}

\section{Conclusions}

The investigation of this specific tautomeric equilibrium support higher stability $\mathbf{A}, \mathbf{B}$ and high energy diradical A' A' ${ }_{\mathbf{a}}, \mathbf{B}^{\prime}, \mathbf{C}^{\prime}$ 'structures in the solution. The presently studied interconversions of the tautomeric forms A' A’ $\mathbf{A}_{\mathbf{a}} \mathbf{B}^{\prime} \mathbf{C}^{\prime}$ confirm pyridine - type nitrogen atoms for N10 N4 N6 and amine type for N4 N3 of the pyridine and 1,3,4 - thiadiazole rings. The intensities of the signals of the NH group in the $100 \mathrm{MHz}{ }^{1} \mathrm{H}-\mathrm{NMR}$ spectra in the range from $8.637 \mathrm{ppm}$ to $7.143 \mathrm{ppm}$ (Tables 10, 11) confirm the mesomeric resonance structures $\mathbf{A}, \mathbf{B}, \mathbf{A}^{\prime} \mathbf{B}^{\prime} \mathbf{C}^{\prime}$ in solution.

\section{Experimental}

\section{General}

Compound 1 was prepared according to the published method [7]. The ${ }^{1} \mathrm{H}-,{ }^{13} \mathrm{C}$ - and ${ }^{15} \mathrm{~N}-\mathrm{NMR}$ measurements of 1 were taken in $\mathrm{CDCl}_{3}$ and in DMSO $-\mathrm{d}_{6}$ solutions, respectively on a Bruker AM 500 spectrometer, operating at $500.18 \mathrm{MHz}$ for hydrogen, $125.76 \mathrm{MHz}$ for carbon and $50.68 \mathrm{MHz}$ for nitrogen, using standard conditions. The $2 \mathrm{D}$ spectra of ${ }^{1} \mathrm{H}-{ }^{13} \mathrm{C} \mathrm{HMQC},{ }^{1} \mathrm{H}-{ }^{13} \mathrm{C}$ HMBC, ${ }^{1} \mathrm{H}-{ }^{1} \mathrm{H}$ COSY (500 MHz) have been recorded in $\mathrm{CDCl}_{3}$ solution according to procedure given in the Bruker programme library. The ${ }^{1} \mathrm{H}-\mathrm{NMR}$ spectra $(1-6)$ of $\mathbf{1}$ were measured on a Tesla BS 677 A spectrometer (100 MHz with T.F.) in $\mathrm{CDCl}_{3}$ or DMSO solutions at room temperature with TMS as the internal standard. The ${ }^{1} \mathrm{H}-\mathrm{NMR}$ spectra $1,1_{3} 1_{4}, 2-6$, $6_{5} 6_{6}(100 \mathrm{MHz})$ and $1_{7}(500 \mathrm{MHz})$ have been recorded in $\mathrm{CDCl}_{3}$ solution and the spectra $1_{1} 1_{2}(100 \mathrm{MHz})$ in DMSO solution $[7,8,10]$. The ${ }^{1} \mathrm{H}-$ 
NMR spectra $1_{1-4}(100 \mathrm{MHz})$ [8] have been taken using various concentration of $\mathbf{1}$ in $\mathrm{DMSO}$ or $\mathrm{CDCl}_{3}$ solutions:

- in a DMSO solution, the concentration of 1 amounts to 1:3 (spectra $1_{1} 1_{2}$, respectively);

- in a $\mathrm{CDCl}_{3}$ solution, the concentration of 1 amounts to: $10 \mathrm{mg} / 0.5 \mathrm{~mL}$ and $25 \mathrm{mg} / 0.5 \mathrm{~mL}$ (maximal concentration, spectra $1_{3} 1_{4}$, respectively).

The ${ }^{1} \mathrm{H}-\mathrm{NMR}$ spectra $1-6,6_{5} 6_{6}$ [7], $1_{7}$ [10] and $1_{8}$ [8] have been recorded in $\mathrm{CDCl}_{3}$ and DMSO $\mathrm{D}_{2} \mathrm{O}$ solutions, respectively, without any determination of the concentration of $\mathbf{1}$. In the ${ }^{1} \mathrm{H}-\mathrm{NMR}$ spectra 1 - 6 of $\mathbf{1}$ the signals of the protons of allyl, pyridyl substituents as well as of $\mathrm{NH}$ group of 1,3,4-thiadiazole have been recorded (Tables 2 - 7, 10,11). In the ${ }^{1} \mathrm{H}$-NMR spectra $6_{5} 6_{6}$ of 1 [7] only the signals of the proton of $\mathrm{NH}$ group of the 1,3,4-thiadiazole have been recorded (Table 11).

The molecular geometries and properties corresponding to the local minima of the energy were calculated at the DFT level of the theory with the B3LYP density functional and the 6-31G* basis set $[24,25]$. The same basis set and functional were used for the ${ }^{1} \mathrm{H}-,{ }^{13} \mathrm{C}$ - and ${ }^{15} \mathrm{~N}-\mathrm{NMR}$ shielding constants calculations by applying the GIAO CPHF methods. The atomic charges were taken from the ESP fit using Breneman model (CHELPG). The Gaussian 98 package [26] was employed for these calculations.

\section{References}

1 Kornis, G. 1,3,4-Thiadiazoles. In Comprehensive Heterocyclic Compounds; Katritzky A.R., Ress W. C., (Eds); Pergamon Press: London. 1984; vol. 6, pp. 545 - 577.

2 deStevens, G.; Eager, M.; Tarby, C. Steric and electronic effects controlling the synthesis of bridgehead nitrogen heterocycles. Heterocycles 1993, 35, 763 - 773.

3 Strzemecka, L. Cyclization reaction of 1,4 - disubstituted thiosemicarbasides. Part II. Pol. J. Chem. 1989, 63, 117 - 123.

4 Strzemecka, L. Cyclization of $\left(\mathrm{N}^{1}\right.$ - cinnamyl- thiocabamyl-)amidrazones. Part II. Pol. J. Chem. 1990, 64, 557 - 566.

5 Strzemecka, L. Otto T., Cyclization reaction of 1,4 - disubstituted thiosemicarbasides. Part I. Pol. J. Chem. 1988, 62, 757 - 766.

6 Strzemecka, L. Cyclization of ( $\mathrm{N}^{1}$-cinnamyl-thiocabamyl)-amidrazones. Part I. Pol. J. Chem. 1990, 64, 157 - 166.

7 Strzemecka, L. Tautomerism of 1,3,4-thiadiazole. Part I. Annales UMCS, Sectio AA 1995/1996, vol. L/LI, 81 - 100.

8 Strzemecka, L. Tautomerism of 1,3,4-thiadiazole. Part II. Annales UMCS, Sectio AA 1999/2000, vol. LIV/LV, 363 -377.

9 Strzemecka, L. Tautomerism of 1,3,4-thiadiazole. Part III. Annales UMCS, Sectio AA 1999/2000, vol. LIV/LV, 379 - 392.

10 Strzemecka, L.; Maciejewska, D.; Urbańczyk-Lipkowska, Z. The structure of N-allyl derivatives of (5-(2'-pyridyl)-[1,3,4]thiadiazol-2-yl) amine in solution and the solid state studied by the ${ }^{1} \mathrm{H}$, ${ }^{13} \mathrm{C},{ }^{15} \mathrm{~N}$ NMR spectroscopy, X-ray crystallography and DFT computations. J. Mol. Struct. 2003, 648, $107-113$. 
11 Fremont, P.; Riverin, H.; Frenette, J.; Rogers, P. A.; Cote, C. Fatigue and recovery of rat soleus muscle are influenced by inhibition of an intracellular carbonic anhydrase isoform. Am. J. Physiol. 1991, 260, 615-21.

12 Kenny, A. D. Role of carbonic anhydraze in bone: plazma acetazolamide concentrations associated with inhibition of bone loss. Pharmacology 1985, 31, 97 - 107.

13 Potts A. C. Stable ophthalmic gel comprising methazolamide. GB 2,223,166, 04 Apr 1990.

14 Miyamoto, K.; Koshiura, R.; Mori, M.; Yokoi, H.; Mori, Ch.; Hasegawa, T.; Takatori, K. Antitumor activity of 5-substituted 2-acylamino-1,3,4-thiadiazoles against transplantable rodent tumors. Chem. Pharm. Bull. 1985, 33, 5126 - 9.

15 Cohen S. M, Ertruk E., Von Esch A.M., Crovetti A.. J., Bryan T. G., Carcinogenicity of 5 nitrofurans and related compounds with amino heterocyclic substituents. J. Natl. Cancer Inst. 1975, 54, 841 - 50

16 Miyahara M., Nakadate M., Sueyohi S., Tanno M., Miyahara M., Kamiya S., Antitumor activity of 2-acylamino-1,3,4-thiadiazoles and related compounds. Chem. Pharm. Bull. 1982, 30, 4402 - 6.

17 Mamolo M. G., Falagiani V., Zampieri D., Vio L., Banfi E., Synthesis and antimycobacterial activity of [5-(pyridin-2-yl)-1,3,4-thiadiazol-2-ylthio]acetic acid arylidene-hydrazide derivatives. Farmaco 2001, 56, 587 - 92.

18 Gadad A. K., Karki S. S., Rajukar V. G., Bhongade B.A., Synthesis and biological evaluation of 5-formyl-6-arylimidazo(2,1-b)-1,3,4-thiadiazole-2-N-(dimethylaminomethino) sulfonamides as antitumor agents. Arzneim. Forsch. 1999, 49, 858 - 63.

19 Cleirci F., Pocar D., Guido M., Loche A., Perlini V, Brufani M., : Synthesis of 2-amino-5sulfanyl-1,3,4-thiadiazole derivatives and evaluation of their antidepressant and anxiolytic activity. J. Med. Chem. 2001, 44, 931 - 6.

20 Barboiu M., Supuran C. T., Menabuoni L., Scozzafawa A., Mincione F., .Briganti F., Mincione G., Carbonic anhydrase inhibitors. Synthesis of topically effective intraocular pressure lowering agents derived from 5-(omega-aminoalkylcarboxamido)-1,3,4-thiadiazole-2-sulfonamide. J. Enzyme Inhib. Med. Chem. 2000, 15, 23 - 46.

21 Mazzone G., Pignatello R., Mazzone S., Panico A., Pennisi G., Castana R., Mazzone P., Synthesis and local anesthetic activity of alkylaminoacyl derivatives of 2-amino-1,3,4-thiadiazole. Farmaco. 1993, 48, 1207 - 24.

22 Cox J. M., Hawkes T. R., Bellini P. E., Russell M., Barrett R., The design and synthesis of inhibitors of imidazoleglycerol phosphate as potential herbicides. Pestic. Sci. 1997, 50, 297 -311.

23 Zucchi F., Trabanelli G., Gonzales N. A., Pyrimidine and thiadiazole derivatives as inhibitors of cooper corrosion in sodium chloride solution. ACH - Mod. Chem. 1995, 132, 579 -88.

24 Lee C., Yang W., Parr R.G. Development of the Colle - Salvetti corelation - energy formula into a functional of the electron density. Phys. Rev. 1988, B 37, 785 - 9.

25 Becke A.D. Density - functional termochemistry. III. The role of exect exchange. J. Chem. Phys. 1993, 98, 5648 - 52.

26 Frisch M.J., Trucks G.W, Schlegel H. B., Scuseria G. E, Robb M. A., Cheeseman J.R., Zakrzewski V.G., Montgomery J.A.Jr, Stratmann R.E., Burant J.C., Dapprich S., Millam J. M, Daniels A. D., Kudin K. N., Strain M. C., Farkas O., Tomasi J., Barone V., Cossi M., Cammi R., Mennucci B., Pomelli C., Adamo C., Clifford S., Ochterski J., Petersson G. A., Ayala P. Y., Cui 
Q., Morokuma K., Malick D. K., Rabuck A. D., .Raghavachari K, Foresman J. B., Cioslowski J., Ortiz J. V., Baboul A.G., Stefanov B. B., Liu G., Liashenko A., Piskorz P., Komaromi I., Gomperts R., Martin L. R., Fox D. J., Keith T., Al-Laham M. A., Peng C.Y., Nanayakkara A., Gonzalez C., Challacombe M., Gill P.M.W., Johnson B., Chen W., Wong M. W., Andres J.L., Gonzalez C., Head-Gordon M., Replogle E. S.; Pople J.A. GAUSSIAN 98, Revision A.7; Gaussian, Inc.: Pittsburgh, PA, 1998.

Sample Availability: Available from the author.

(C) 2006 by MDPI (http://www.mdpi.org). Reproduction is permitted for noncommercial purposes. 